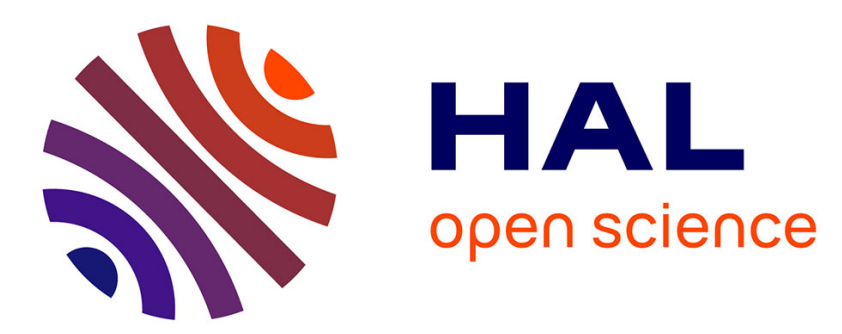

\title{
Les femmes au chômage retardent l'arrivée du premier enfant
}

\author{
Monique Meron, Blöss-Widmer Isabelle
}

\section{To cite this version:}

Monique Meron, Blöss-Widmer Isabelle. Les femmes au chômage retardent l'arrivée du premier enfant. Population (édition française), 2002, 57 (2), pp.327-357. 10.3917/popu.202.0327 . halshs-01272500

\section{HAL Id: halshs-01272500 \\ https://shs.hal.science/halshs-01272500}

Submitted on 11 Feb 2016

HAL is a multi-disciplinary open access archive for the deposit and dissemination of scientific research documents, whether they are published or not. The documents may come from teaching and research institutions in France or abroad, or from public or private research centers.
L'archive ouverte pluridisciplinaire HAL, est destinée au dépôt et à la diffusion de documents scientifiques de niveau recherche, publiés ou non, émanant des établissements d'enseignement et de recherche français ou étrangers, des laboratoires publics ou privés. 


\title{
LES FEMMES AU CHÔMAGE RETARDENT L'ARRIVÉE DU PREMIER ENFANT
}

\author{
Monique Meron, Isabelle Widmer
}

Institut national d'études démographiques (INED) | « Population »

2002/2 Vol. 57 | pages 327 à 357

ISSN 0032-4663

Article disponible en ligne à l'adresse :

http://www.cairn.info/revue-population-2002-2-page-327.htm

\section{!Pour citer cet article :}

Monique Meron, Isabelle Widmer, «Les femmes au chômage retardent l'arrivée du premier enfant », Population 2002/2 (Vol. 57), p. 327-357.

DOI 10.3917/popu.202.0327

Distribution électronique Cairn.info pour Institut national d'études démographiques (INED).

(C) Institut national d'études démographiques (INED). Tous droits réservés pour tous pays.

La reproduction ou représentation de cet article, notamment par photocopie, n'est autorisée que dans les limites des conditions générales d'utilisation du site ou, le cas échéant, des conditions générales de la licence souscrite par votre établissement. Toute autre reproduction ou représentation, en tout ou partie, sous quelque forme et de quelque manière que ce soit, est interdite sauf accord préalable et écrit de l'éditeur, en dehors des cas prévus par la législation en vigueur en France. Il est précisé que son stockage dans une base de données est également interdit. 


\title{
Les femmes au chômage retardent l'arrivée du premier enfant
}

\author{
Monique MERON* et Isabelle WIDMER**
}

\begin{abstract}
Dans nombre de pays développés, le retard dans la constitution des familles est lié à la sortie plus tardive du système scolaire et au souhait de plus en plus fréquent des femmes de commencer leur carrière professionnelle avant d'avoir des enfants (en France, par exemple, le taux d'activité des femmes de 25 à 29 ans dépasse $80 \%$ ). Mais qu'advient-il quand elles rencontrent des difficultés d'insertion professionnelle, en particulier des périodes de chômage? Pour tenter de répondre à cette question, Monique MERON et Isabelle WIDMER ont utilisé des données biographiques professionnelles et familiales collectées dans une enquête nationale. Il apparaît que des périodes de chômage au début de la vie en couple conduisent à retarder encore l'arrivée du premier enfant. Les femmes touchées par le chômage ont des comportements familiaux très différents des femmes au foyer qui, elles, ont un premier enfant plus rapidement que celles qui sont sur le marché du travail.
\end{abstract}

Quels liens existent entre l'évolution des comportements familiaux et les transformations du marché du travail? On sait que la conjoncture économique a un impact particulièrement important sur les débuts de la vie professionnelle des jeunes au moment où ils sont susceptibles de construire une vie de famille. Mais comment va réagir une jeune femme qui vit en couple sans enfant lorsqu'elle est confrontée à une période de chômage ? Va-t-elle avancer son éventuel projet de maternité à la faveur du temps « libre » qui lui est ainsi imposé ? Ou bien, au contraire, y renoncerat-elle temporairement devant l'incertitude sur l'avenir et les difficultés matérielles liées à la recherche d'un emploi ? À moins que ces deux sphères - familiale et professionnelle - ne restent parfaitement indépendantes l'une de l'autre? Au regard du lien avec la fécondité, une période

\footnotetext{
* Institut national d'études démographiques.

** Département de sociologie, université d'Aix-Marseille I; Institut national d'études démographiques.

Cet article résulte d'un travail financé par la Direction de la population et des migrations (DPM) suite à une recommandation du Haut Conseil de la population et de la famille (rapport de Jacques Commaille).
} 
de chômage est-elle ou non assimilable à une période d'inactivité ? Le comportement des chômeuses est-il plutôt proche de celui des femmes au foyer ou de l'attitude de celles qui ont un emploi?

Depuis le milieu des années 1970, l'âge à la première maternité recule tandis que les taux de chômage, tout particulièrement ceux des jeunes femmes, se sont considérablement accrus. Le risque de chômage comme le calendrier de la fécondité dépendent de la place des femmes dans la société, de la génération à laquelle elles appartiennent et de la formation qu'elles ont reçue; mais les statistiques descriptives habituelles ne permettent pas d'établir un lien clair entre les fluctuations du chômage et l'âge de la mère à la venue du premier enfant, même en tenant compte de ces différences.

Les méthodes utilisées ici s'appuient sur des itinéraires biographiques; elles permettent d'analyser les trajectoires individuelles dans toute leur diversité pour mieux approcher l'interaction entre le contexte d'activité des femmes et la décision d'avoir ou non un premier enfant. Cette approche biographique est appliquée aux femmes interrogées dans l'enquête Jeunes et carrières réalisée en 1997 par l'Insee. Dans cette enquête représentative au niveau national, on dispose d'un large échantillon de femmes ayant ou non vécu en couple. Pour des raisons d'homogénéité des données et de recul suffisant par rapport au début de la période de vie féconde, on examinera d'abord les calendriers des femmes âgées de 31 à 45 ans en 1997 (nées de 1952 à 1966); puis on se demandera si les tendances se prolongent parmi les plus jeunes, âgées de 24 à 29 ans (nées de 1968 à 1973).

Débuts dans la vie familiale et entrée dans la vie active : des calendriers bousculés

Les générations féminines les plus récentes ont franchi plus tard que leurs aînées les étapes considérées comme constitutives du passage à la vie adulte (fin des études, départ de chez les parents, début d'une vie en couple, recherche d'un emploi); leurs calendriers, bien que plus serrés et plus précoces que ceux des hommes, n'ont cessé de s'allonger depuis le milieu des années 1970 (Galland, 1995, 2000; Galland et Meron, 1996).

En parallèle, l'âge moyen des femmes à la maternité est passé de près de 27 ans en 1975 à plus de 29 ans en 2000 (Prioux, 2001). Ce recul a eu lieu, pour l'instant, sans remettre en cause le renouvellement des générations puisque toutes ont eu environ 2,1 enfants par femme à 40 ans.

En ce qui concerne la première maternité, l'âge moyen des femmes au moment de l'accouchement est passé d'un minimum de moins de 24 ans en 1972 à 25 ans en 1983, puis 26 ans en 1989.

«Ainsi, à leur vingt-cinquième anniversaire, seules $37 \%$ des femmes de la génération née en 1965 étaient déjà mères, au lieu de $55 \%$ dans la génération 1955, et $61 \%$ dans la génération 1945. »(Prioux, 1996, p. 16) 
Depuis, l'âge auquel la moitié des femmes sont devenues mères est évalué, d'après les enquêtes de l'Insee, à 26 ans pour celles qui sont nées de 1963 à 1966 et à 28,2 ans pour les femmes nées de 1968 à 1971 (Galland, 2000).

La venue plus tardive du premier enfant est liée aux transformations de la famille, à l'allongement des études et au développement de l'activité féminine

À partir des années 1970, la durée de vie en couple avant la venue du premier enfant s'est allongée, inversant les tendances de la décennie précédente. Les démographes expliquent cette évolution par la diffusion des nouvelles méthodes de contraception en France, la meilleure maîtrise de la fécondité contribuant à l'allongement de tous les intervalles génésiques. Cependant, si le recul de l'âge à la première maternité observé au milieu des années 1970 pouvait être clairement attribué au recours croissant à une contraception moderne et efficace, la poursuite de ce mouvement demande d'autres explications.

La fragilisation des relations de couple et l'augmentation de la cohabitation hors mariage ont aussi marqué l'époque récente; dans ce contexte, le fait que $83 \%$ des enfants vivent avec leurs deux parents en 1994 (Villeneuve-Gokalp, 1999) indique que les enfants sont aujourd'hui, dans leur immense majorité, le résultat d'un projet de couple mûrement réfléchi (Toulemon, 1994).

Depuis la fin de la seconde guerre mondiale, l'allongement de la durée des études en France a été spectaculaire puisque cette durée a doublé en cinquante ans. La part de bacheliers dans une génération est passée de $4 \%$ en 1946 à plus de $60 \%$ aujourd'hui et la proportion de titulaires de diplômes techniques et professionnels (CAP, BEP) parmi les 25-34 ans a triplé sur la période (Estrade et Minni, 1996). Les femmes ont particulièrement bénéficié de cette évolution : depuis 1996, la durée de leurs études est désormais plus longue que celle des hommes et elles ont un niveau de qualification plus élevé, ce qui était loin d'être le cas de leurs aînées.

Or, l'âge à la première maternité s'élève avec le niveau de formation des femmes : à la naissance de leur premier enfant, les femmes nées durant les années 1950 à 1954 avaient en moyenne 22,6 ans si elles étaient titulaires du seul certificat d'études primaires et 28,4 ans lorsqu'elles avaient un diplôme de niveau bac +4 . Les écarts selon le niveau de diplôme et le milieu social se sont accrus au cours des années 1980 et 1990 (Desplanques et de Saboulin, 1986). D'après les prévisions, les femmes des générations 1960-1964 titulaires de diplômes de niveau bac +4 auront, en moyenne, encore retardé de 1,5 année la venue du premier enfant par rapport à celles nées dix ans plus tôt. En revanche, l'âge moyen à la première maternité des femmes peu diplômées resterait quasi stable. F. de Singly explique comment « les femmes ayant des perspectives professionnelles repoussent leur mariage, le temps des études et d'un premier emploi » (1987, p. 182); «par ailleurs, tant qu'elle poursuit des études 
supérieures, une femme évite d'avoir des enfants, ce qui conduit à des naissances plus tardives »(Desplanques, 1996, p. 17). Le recul général de l'âge à la première naissance est donc d'autant plus accusé d'une génération à la suivante que les femmes sont de plus en plus nombreuses à être diplômées.

L'activité féminine n'a cessé de se développer depuis le début des années 1970 : près de $80 \%$ des femmes âgées de 25 à 49 ans ont ou recherchent un emploi (à temps plein ou à temps partiel) en mars 2001 alors qu'elles étaient moins de 59 \% en 1975 (Aerts et Mercier, 2001). Mais le taux d'activité des femmes est d'autant plus faible que le nombre de leurs enfants augmente. En janvier 1999, $79 \%$ des femmes âgées de 25 à 49 ans étaient actives; ce taux était de $88 \%$ parmi les femmes en couple sans enfant, $83 \%$ pour les mères d'un enfant, $74 \%$ pour celles qui avaient deux enfants et $52 \%$ pour les mères d'au moins trois enfants. À l'exception d'une diminution très récente due à l'influence de l'extension de l'allocation parentale d'éducation en juillet 1994 (Bonnet et Labbé, 1999), c'est l'activité des mères de deux enfants et plus qui a le plus progressé.

Chez les plus jeunes, l'inactivité (au sens du retrait par rapport au marché du travail) est devenue rare : en 1995, $8 \%$ des femmes de 15 à 29 ans étaient au foyer alors qu'elles étaient $19 \%$ en 1975 (Meron et Minni, 1995). Depuis 1995, certaines évolutions ont cependant marqué le pas : la durée des études a cessé de s'allonger et l'inactivité féminine ne diminue plus (Brunet et Minni, 2000).

L'effet de la situation professionnelle des femmes est peu étudié en dehors des préoccupations portant sur le nombre final d'enfants; en particulier, l'incidence des différents types d'activité (emploi, chômage, inactivité) sur le calendrier familial des femmes n'est pas établie. Des sociologues et des économistes de la famille ont cependant développé l'idée que les sphères familiale et professionnelle ne sont pas dissociées et se situent dans une logique de division sexuelle du travail (BarrèreMaurisson et Marchand, 2000).

\section{Difficultés d'insertion et débuts familiaux plus tardifs : quels liens?}

La crise économique s'est traduite pour les jeunes par des difficultés d'insertion. Le chômage, qui s'est largement accru depuis la fin des années de croissance, les frappe tout particulièrement et touche le plus durement les femmes (Maruani, 1998). Les taux de chômage, qui se maintenaient aux alentours de $2,5 \%$, se sont envolés dès la première moitié des années 1970 pour dépasser $10 \%$ en 1985. Après une courte embellie à la fin des années 1980, le chômage a atteint des niveaux records en 1994 puis en 1997, dépassant $12 \%$ de la population active, avant d'amorcer une nouvelle diminution depuis 1998 . En mars 2001 , on comptait $8,8 \%$ de chômeurs parmi l'ensemble des personnes présentes sur le marché du travail, et les taux de chômage, encore très différenciés selon le niveau de formation, s'échelonnaient de $14,1 \%$ pour ceux qui ne possédaient aucun 
diplôme ou seulement le certificat d'études primaires, à environ $5 \%$ pour les titulaires d'un diplôme supérieur (Aerts et Mercier, 2001).

Bien que les jeunes poursuivent de plus en plus longtemps leurs études, près d'un sur deux (48\%) est présent sur le marché du travail entre 15 et 29 ans. Malgré l'amélioration de ces dernières années, le taux de chômage des jeunes reste très élevé et leurs emplois sont particulièrement sensibles aux fluctuations de la conjoncture, surtout pour les moins diplômés et pour les derniers arrivés sur le marché du travail (Brunet et Minni, 2000 ; Fondeur et Minni, 1999; Meron et Minni, 1995).

Ainsi, le contexte dans lequel les jeunes d'aujourd'hui s'insèrent dans la vie professionnelle et adulte est beaucoup plus difficile que du temps de leurs parents; les perspectives d'avenir des jeunes adultes, notamment en termes de carrières salariales, en sont bouleversées (Baudelot et Establet, 2000), ce qui n'est certainement pas sans influence sur la constitution des familles. La précarité est parfois évoquée comme facteur explicatif du maintien de la cohabitation avec les parents et du recul du moment de fonder une famille, surtout chez les jeunes hommes (Leridon et Villeneuve-Gokalp, 1994; Galland et Meron, 1996; Dormont et Dufour-Kippelen, 2000). L'influence exercée par les facteurs économiques sur la mise en couple a aussi été étudiée : parmi les générations les plus récentes (nées après 1968), "l'effet du “niveau de précarité" n'est pas identique pour les hommes et les femmes. C'est ainsi qu'un contrat à durée déterminée n'est pas pénalisant pour les femmes alors qu'il l'est pour l'homme. Mais une situation de précarité plus grande (contrats aidés, stage d'insertion, stage non rémunéré, chômage) est un facteur négatif pour les deux sexes » (Ekert et Solaz, 2000; Kieffer, Marry, Meron et Solaz, à paraître en 2002).

\section{Monographies et enquêtes transversales apportent des réponses contradictoires à l'impact du chômage sur les projets d'enfant}

Dans les travaux en sciences sociales, le chômage apparaît tantôt comme un facteur incitant à remettre à des temps meilleurs les projets de fécondité, tantôt comme un temps de «non-activité » pouvant être mis à profit pour avoir un enfant.

Ainsi, d'après les derniers résultats de l'enquête permanente sur les conditions de vie de 1998, «72\% des femmes de moins de 25 ans jugent "très important" pour une femme le fait d'avoir un travail stable avant d'avoir un premier enfant» (Toulemon et Leridon, 1999). Mais d'autres études, fondées sur des récits de vies de jeunes femmes en situation précaire, accréditent l'idée que, confrontées aux difficultés d'insertion, les jeunes femmes ont tendance à se replier sur la sphère familiale.

«Ces jeunes femmes vont "utiliser" leur précarité professionnelle en mettant en œuvre des projets dans les "entractes" que provoquent les turbulences d'emploi (ces projets vont de la mobilité volontaire à la formation en passant par la "programmation" d'un enfant). » (Nicole-Drancourt et Roulleau-Berger, 1995, p. 79; Nicole-Drancourt, 1989) 
Or, dans les récits de vies, la proximité entre une période de chômage et la venue d'un enfant peut amener l'enquêtée à reconstruire, en toute bonne foi, une explication a posteriori. Les entretiens biographiques constituent des manières «d'inventer des stratégies discursives pour faire face à la situation d'entretien, pour "garder la face" (Goffman, 1974) et affirmer leur spécificité de sujet » (Demazière et Dubar, 1997).

Si le chômage et l'âge à la première maternité ont tous deux tendance à augmenter depuis le milieu des années 1970, la comparaison entre l'évolution de la conjoncture économique et celle du calendrier des événements familiaux ne permet pas d'établir un lien direct entre chômage et fécondité (figures 1 et 2) : bien que moins touchées par le chômage, les femmes les plus diplômées retardent particulièrement la venue du premier enfant; au contraire, les moins qualifiées ont des enfants plus tôt, alors qu'elles sont plus sensibles à la conjoncture économique. De plus, aucune fluctuation similaire à celles des courbes de chômage n'est observée sur les courbes d'âge à la maternité, quel que soit le niveau de qualification des femmes, même avec un décalage dans le temps.

Devant les conclusions contradictoires apportées par les enquêtes d'opinion, les monographies et la confrontation des données statistiques à propos de l'impact du chômage sur les projets d'enfants, une approche biographique a été envisagée.

\section{L'analyse biographique permet d'aborder le sujet différemment}

Les conséquences du chômage sur la vie familiale sont multiples et diversifiées : elles dépendent non seulement de la place du chômeur dans le ménage et du type de chômage auquel il est confronté mais aussi du moment où la période de chômage intervient dans la vie de l'individu. Elles varient donc notamment selon l'âge, le milieu d'origine, la situation du conjoint, l'ancienneté du couple... Pour mesurer l'impact du chômage sur la première maternité des femmes, on a étudié les durées passées en couple sans enfant à l'aide d'une analyse des biographies (Courgeau et Lelièvre, 1989).

L'enquête Jeunes et carrières de l'Insee (Estrade et Thiesset, 1998) se prête à ce type d'analyse car elle décrit dans un calendrier annuel(1) rétrospectif tous les événements familiaux, résidentiels et professionnels concernant l'individu depuis l'année de ses 16 ans (annexe, section 1).

Les femmes sont observées à partir du début de leur première vie en couple jusqu'à la naissance de leur premier enfant, ou jusqu'à la séparation de leur premier conjoint si celle-ci intervient avant la naissance d'un enfant, ou jusqu'à la date de l'enquête sinon; on se limite à un maximum de huit ans de vie commune.

(1) Dans les trajectoires observées, l'année est l'unité de temps; des travaux antérieurs utilisant la même méthode ont montré que les dates mensuelles n'apportent à elles seules que peu d'information supplémentaire (Kempeneers et Lelièvre, 1991). 


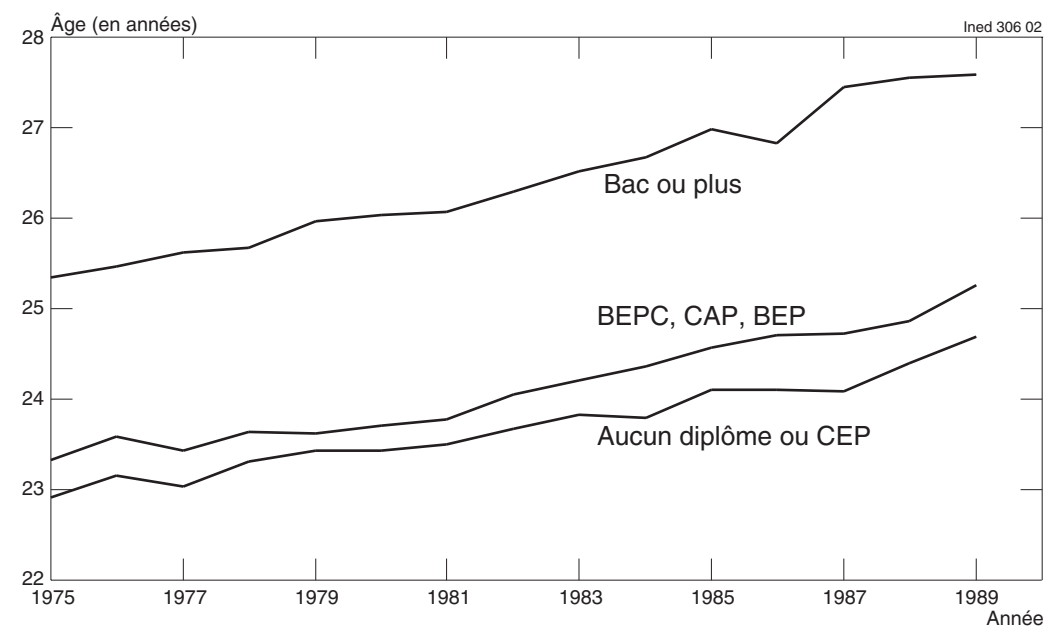

Figure 1.- Âge moyen des mères à la naissance du premier enfant selon le niveau de diplôme

Source: Insee, enquête Famille 1990.

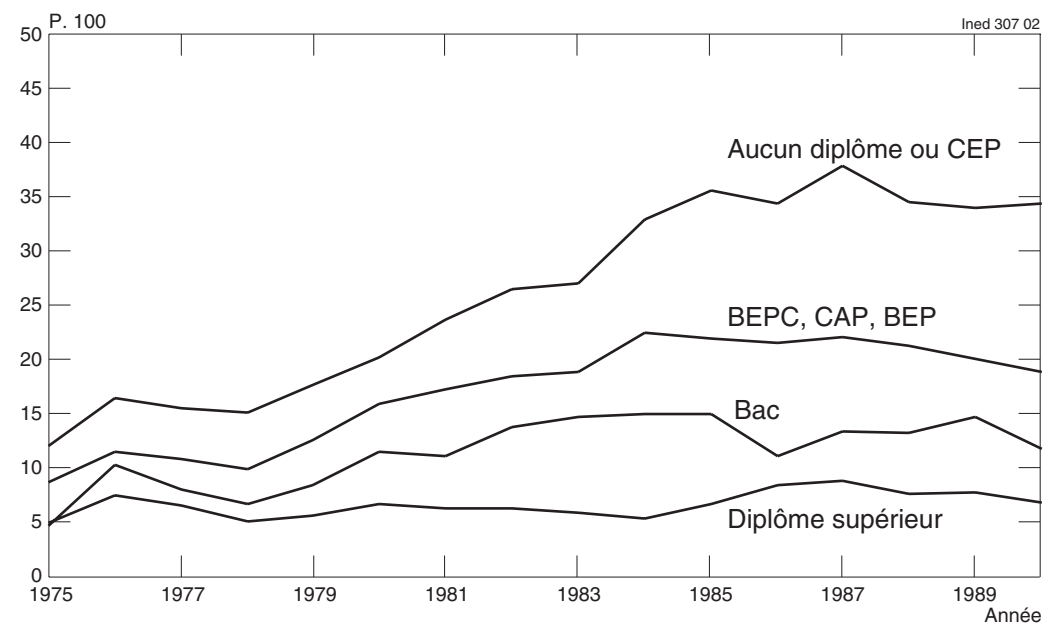

Figure 2.- Taux de chômage des femmes de 15 à 29 ans selon le niveau de diplôme

Source: Insee, enquêtes Emploi. 
Dans un premier temps, on s'intéressera ici aux femmes de 31 à 45 ans à la date de l'enquête (1997), qui ont mentionné au moins une date de mise en couple ou de mariage dans le calendrier rétrospectif. On a éliminé celles qui ont eu un premier enfant avant l'année de la mise en couple. En adaptant les variables aux plus jeunes (et en se limitant aux 2429 ans), on cherchera ensuite à savoir si les tendances dégagées se poursuivent avec les générations plus récentes.

\section{Une femme sur deux, née de 1952 à 1966, a eu un premier enfant lors de ses trois premières années de vie conjugale}

Le premier fichier constitué comprend 5506 femmes nées de 1952 à 1966 dont 877 (16\% de données dites « interrompues à droite ») n'ont pas eu d'enfant dans la fenêtre observée. Elles sont représentatives de 5660000 femmes âgées de 31 à 45 ans en 1997. Une sur deux a eu un enfant lors de ses trois premières années de vie conjugale (annexe, section 2 et tableau A).

La durée de vie en couple sans enfant dépend de caractéristiques propres à la femme (génération à laquelle elle appartient, niveau de diplôme, etc.) mais aussi des événements qu'elle a pu rencontrer pendant cette période et du contexte dans lequel elle vit. On a pu tenir compte de l'origine sociale et des variables relatives aux situations vécues vis-à-vis des études et du marché du travail. Mais d'autres variables manquent dans l'enquête. Ainsi, les caractéristiques du premier conjoint ou les conditions de logement n'apparaissent pas explicitement dans l'analyse alors qu'elles jouent aussi un rôle dans les calendriers de fécondité (annexe, section 3). Par ailleurs, la situation de chômage est peut-être corrélée avec une certaine instabilité conjugale; toutefois, le fait de prendre en compte la date de séparation du premier conjoint lorsque celle-ci intervient dans la fenêtre observée contribue à réduire cet effet. Il est logique d'arrêter alors l'observation puisqu'une séparation va, selon toute vraisemblance, changer le comportement de la femme vis-à-vis de son calendrier de fécondité.

Les situations d'activité sont repérées dans le calendrier annuel de la femme pendant la vie en couple qui précède l'arrivée du premier enfant, c'est-à-dire à partir du début de la vie conjugale jusqu'à l'année antérieure à la naissance du premier enfant pour celles qui en ont eu un, ou jusqu'à la date de fin d'observation pour les autres (annexe, section 2). C'est donc bien l'influence du contexte économique sur la date de naissance du premier enfant qui est étudiée, sans risquer d'interférer avec l'influence inverse de la venue de l'enfant sur la situation professionnelle de la femme (on ne tolère ce flou que dans le cas où naissance et début de vie en couple ont lieu la même année). Lors de l'enquête, les périodes de chômage, d'inactivité et d'emploi long ne sont repérées que si elles ont duré au moins 6 mois. Une période d'emploi «intermittent » désigne des emplois plus courts alternant avec des périodes sans emploi. On a regroupé études et reprises d'études dans une même rubrique «études ». Ces situations ne 
sont pas nécessairement exclusives : on peut en rencontrer jusqu'à trois (consécutives ou concomitantes) mentionnées pour une même année. Toutes les déclarations comptent de la même façon dans l'analyse, et n'ont pas été hiérarchisées a posteriori.

\section{Les plus jeunes et les plus diplômées sont restées plus longtemps en couple sans enfant...}

Si la moitié des femmes en couple nées entre 1952 et 1966 ont eu un premier enfant durant les 3 premières années de vie conjugale, cette durée médiane n'est que de 2,1 ans pour celles qui n'ont pas de diplôme ou seulement un certificat d'études primaires, alors qu'elle atteint 3,7 ans pour les bachelières et 4,7 ans pour les titulaires d'un diplôme de niveau supérieur à $\mathrm{bac}+2$.

Par ailleurs, parmi les générations 1952 à 1954, une femme sur deux a attendu moins de 2,4 ans après le début de sa vie en couple pour avoir un premier enfant alors que parmi les plus jeunes (nées de 1964 à 1966), cet intervalle est une fois et demie plus long : 3,7 ans (tableau A). Entre ces deux extrêmes, la durée médiane s'allonge régulièrement d'une génération à la suivante.

La durée de la vie conjugale sans enfant dépend donc à la fois de la génération et du niveau de diplôme, qui résume en quelque sorte le milieu social de la jeune femme (Galland, 1995). Dans les analyses non paramétriques, la fonction de séjour (ou de survie) indique la proportion de femmes qui, pour une durée de vie en couple donnée, sans sortie d'observation, n'ont pas encore eu d'enfant. Ces courbes illustrent bien le fait que plus les femmes sont jeunes et diplômées, plus elles vivent longtemps en couple avant d'avoir un premier enfant (figures 3 et 4).

Pour examiner l'influence d'autres variables, on se heurte vite au problème de l'insuffisance des effectifs concernés dans le sondage : on sera donc amené, dans certaines analyses, à résumer les effets précédents en regroupant les niveaux de diplômes - bachelières ou non-bachelières et les générations - on distinguera les femmes nées dans les années 1950 (c'est-à-dire de 1952 à 1960) et les femmes nées dans les années 1960 (c'est-à-dire de 1961 à 1966) - (tableau 1).

Afin de contrôler la liaison entre niveau de diplôme et génération, une analyse semi-paramétrique (modèle de Cox) a été réalisée. Elle permet d'étudier la durée passée en couple sans enfant, compte tenu des variables explicatives introduites. Le modèle de Cox appartient à la famille des modèles à risques proportionnels et présente l'intérêt d'estimer directement l'effet de la durée, sans lui imposer de loi a priori, bien qu'il suppose un effet proportionnel des caractéristiques individuelles des femmes sur les risques d'avoir un premier enfant. Son utilisation requiert donc uniquement une validation (graphique) du parallélisme des courbes représentant les logarithmes des quotients cumulés en fonction de la 


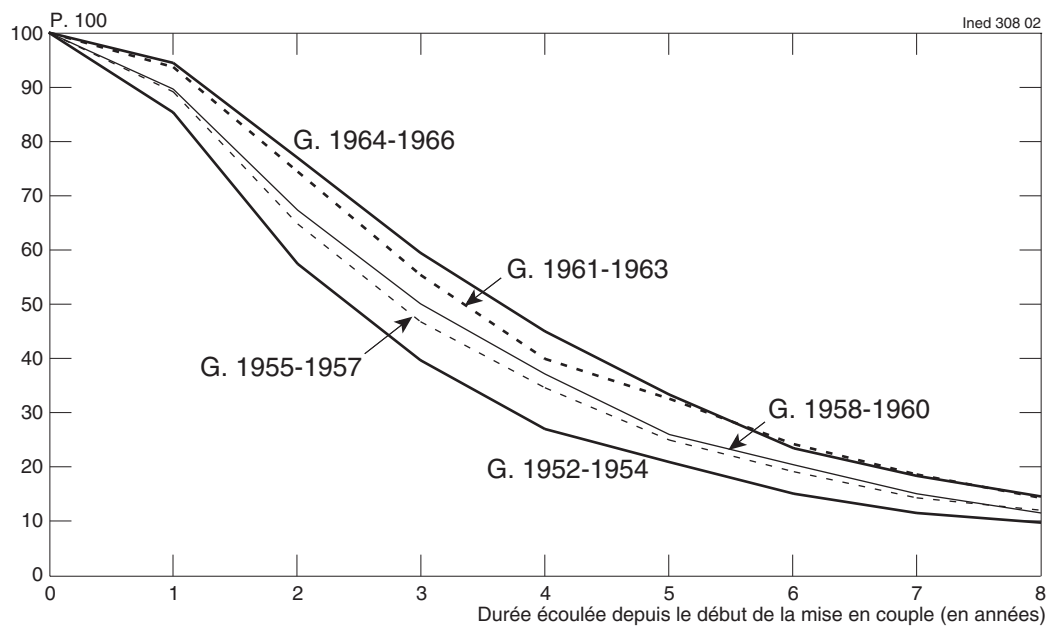

Figure 3.- Proportion de femmes sans enfant à chaque durée de vie en couple selon la génération (fonction de séjour)

Source : Insee, enquête Jeunes et carrières 1997.

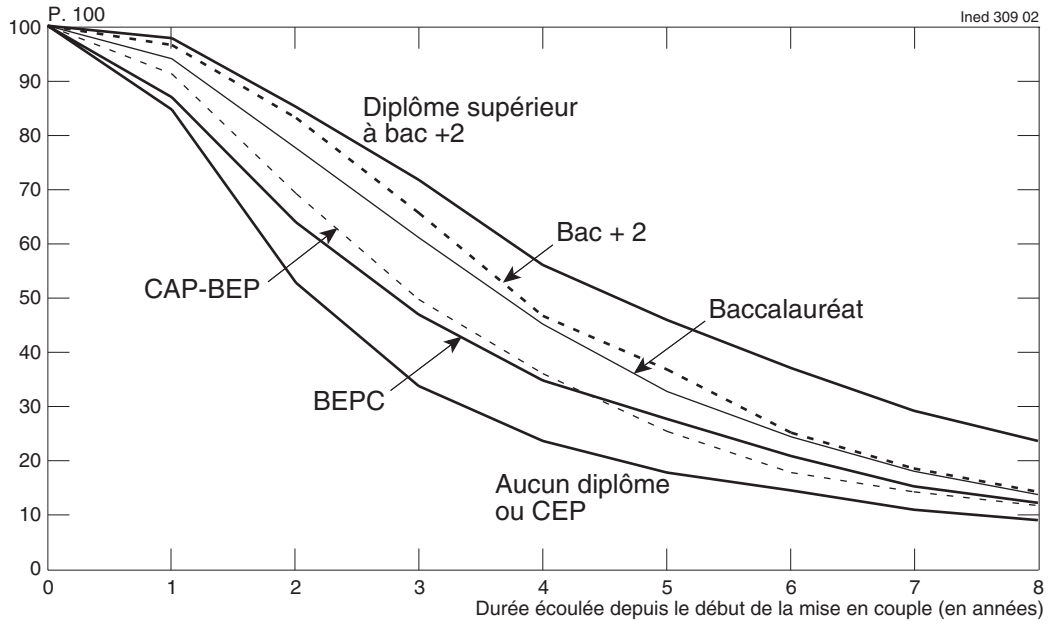

Figure 4.- Proportion de femmes sans enfant à chaque durée de vie en couple selon le niveau de diplôme (fonction de séjour)

Source: Insee, enquête Jeunes et carrières 1997. 


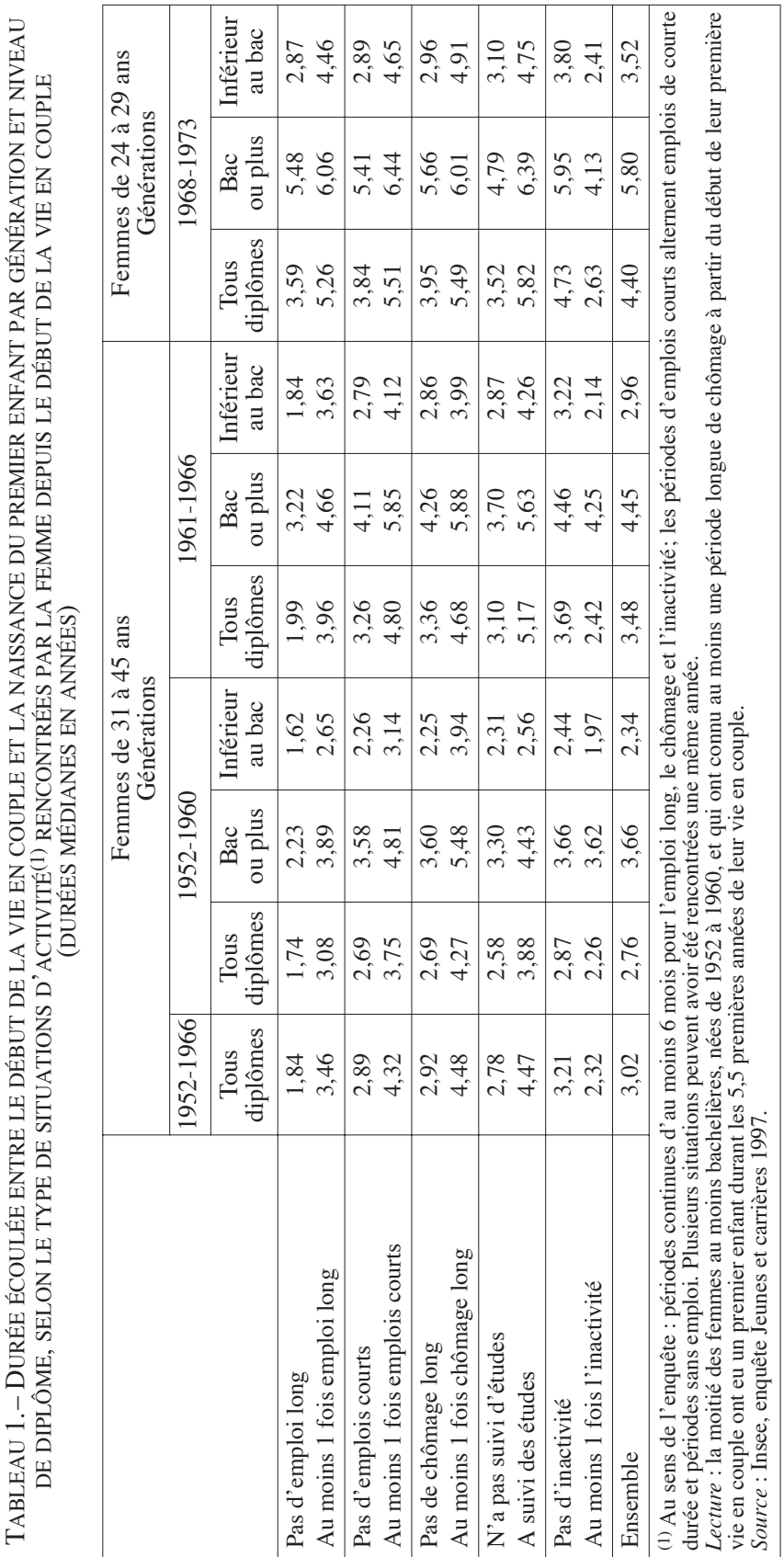


durée(2). Dans une telle analyse, les courbes se déduisent les unes des autres en fonction d'un facteur multiplicatif; ces coefficients, qui s'interprètent par rapport à 1 , sont présentés dans les tableaux de résultats des modèles. Cette analyse confirme que les différences dues à chacune des deux variables (niveau de diplôme et génération) restent significatives lorsqu'on les fait intervenir ensemble.

\section{... mais cela ne dépend pas de l'âge auquel a débuté la vie en couple}

Pour les femmes nées entre 1963 et 1966, l'âge médian au début de la vie conjugale va de 20,2 ans pour celles qui ont un diplôme inférieur au CAP à 21,8 ans pour les titulaires d'un CAP-BEP, 23,1 ans pour les bachelières et 24,4 ans pour les femmes diplômées du supérieur. Or, l'analyse montre que cet âge au moment de la mise en couple ne joue pas de rôle significatif, une fois que l'on a tenu compte des deux premières variables considérées, c'est-à-dire la génération et le niveau de diplôme (tableau 2).

TABLEAU 2.- DURÉE ÉCOULÉE ENTRE LE DÉBUT DE LA VIE EN COUPLE ET LA NAISSANCE DU PREMIER ENFANT EN FONCTION DE LA GÉNÉRATION, DU NIVEAU DE DIPLÔME ET DE L'ÂGE AU DÉBUT DE LA VIE EN COUPLE (ANALYSE SEMI-PARAMÉTRIQUE) - ÉCHANTILLON DES FEMMES DE 31 À 45 ANS

\begin{tabular}{|c|c|c|}
\hline & Paramètre & Effet multiplicatif \\
\hline \multicolumn{3}{|l|}{ Génération } \\
\hline $1952-1954$ & $0,15 * *$ & 1,16 \\
\hline 1955-1957 & 0,02 & 1,02 \\
\hline 1958-1960 & Réf. & 1,00 \\
\hline $1961-1963$ & $-0,13 *$ & 0,88 \\
\hline 1964-1966 & $-0,16 * * *$ & 0,85 \\
\hline \multicolumn{3}{|l|}{ Niveau de diplôme } \\
\hline Supérieur à bac + 2 & $-0,46^{* * *}$ & 0,63 \\
\hline $\mathrm{Bac}+2$ & $-0,22 * * *$ & 0,80 \\
\hline $\mathrm{Bac}$ & $-0,17 * * *$ & 0,84 \\
\hline BEPC, CAP, BEP & Réf. & 1,00 \\
\hline Sans diplôme ou CEP & $0,20 * * *$ & 1,22 \\
\hline \multicolumn{3}{|l|}{ Âge à la mise en couple } \\
\hline $16-19$ ans & 0,07 & 1,07 \\
\hline $20-21$ ans & $-0,06$ & 0,94 \\
\hline $22-24$ ans & Réf. & 1,00 \\
\hline 25 ans ou plus & $-0,03$ & 0,97 \\
\hline \multicolumn{3}{|c|}{$\begin{array}{l}\text { * Significatif au seuil de } 5 \% \text {; } * \text { significatif au seuil de } 5 \% \text {; } * * * \text { significatif au seuil de } 1 \% \text {. } \\
\text { Lecture: toutes choses égales par ailleurs, les femmes nées de } 1952 \text { à } 1954 \text { ont } 1,16 \text { fois plus de chances } \\
\text { d'avoir eu un premier enfant depuis le début de leur vie en couple que les femmes nées de } 1958 \text { à } 1960 \\
\text { (référence). } \\
\text { Source: Insee, enquête Jeunes et carrières } 1997 \text {. }\end{array}$} \\
\hline
\end{tabular}

(2) Cette condition a été vérifiée pour les 8 premières années de vie conjugale, mais les graphiques ne sont pas présentés dans cet article. 
Ce résultat, assez étonnant, indique toutefois que ces variables, en résumant les caractéristiques du passé et du milieu social de la jeune femme, permettent de bien expliquer l'âge au moment de la mise en couple. Il est donc ici inutile de faire intervenir cet âge dans l'analyse, en plus de la génération et du niveau de diplôme.

En revanche, d'autres variables, connues pour être déterminantes dans les comportements de fécondité, exercent bien ici une influence significative. Ainsi, à niveau de diplôme et génération donnés, les femmes provenant de familles nombreuses deviennent mères plus précocement que la moyenne et l'on sait qu'elles ont un nombre d'enfants en moyenne plus élevé que les femmes issues de familles restreintes ( 1 ou 2 enfants) (Leridon, 1985, p. 517). Par ailleurs, les femmes dont le père est ouvrier ou la mère inactive ont plus rapidement un premier enfant que celles dont la mère ou le père est cadre. Enfin, les femmes qui habitent en milieu rural ou des villes de moins de 20000 habitants ont un premier enfant un peu plus vite que les habitantes des plus grandes villes ou de Paris.

\section{Les femmes confrontées au chômage ont plus retardé l'arrivée d'un premier enfant...}

Les trois quarts des femmes nées de 1952 à 1966 ont occupé au moins un emploi de six mois ou plus sur la période observée (c'est-à-dire entre le début de la première vie conjugale et l'année précédant l'arrivée d'un premier enfant ou l'année de fin d'observation). L'existence de périodes d'emploi intermittent et de chômage est surtout le lot des plus jeunes et des moins diplômées. L'exigence de l'enquête pour repérer le chômage (au moins 6 mois continus de recherche d'emploi) explique la relative rareté de cette situation, qui concerne tout de même près d'une femme sur dix dans ces générations. La poursuite d'études après le début de la vie en couple est le fait d'une femme sur cinq, et s'observe surtout chez les plus diplômées (plus d'une sur trois). Ce sont plus souvent les moins qualifiées (celles qui n'avaient pas le baccalauréat) qui ont signalé une période d'au moins six mois d'inactivité (plus d'une sur cinq) (tableau B, annexe, section 2).

Par rapport aux femmes de la même génération et de même niveau de diplôme, celles qui ont toujours été présentes sur le marché du travail ont eu un premier enfant plus longtemps après le début de leur vie en couple. Mais cet allongement de la durée de vie en couple sans enfant est plus important pour celles qui ont connu des périodes d'emploi intermittent que pour celles qui décrivent seulement des périodes d'emploi continu, et ce sont les périodes continues de chômage qui retardent le plus l'arrivée du premier enfant (tableau 1, figure 5). 

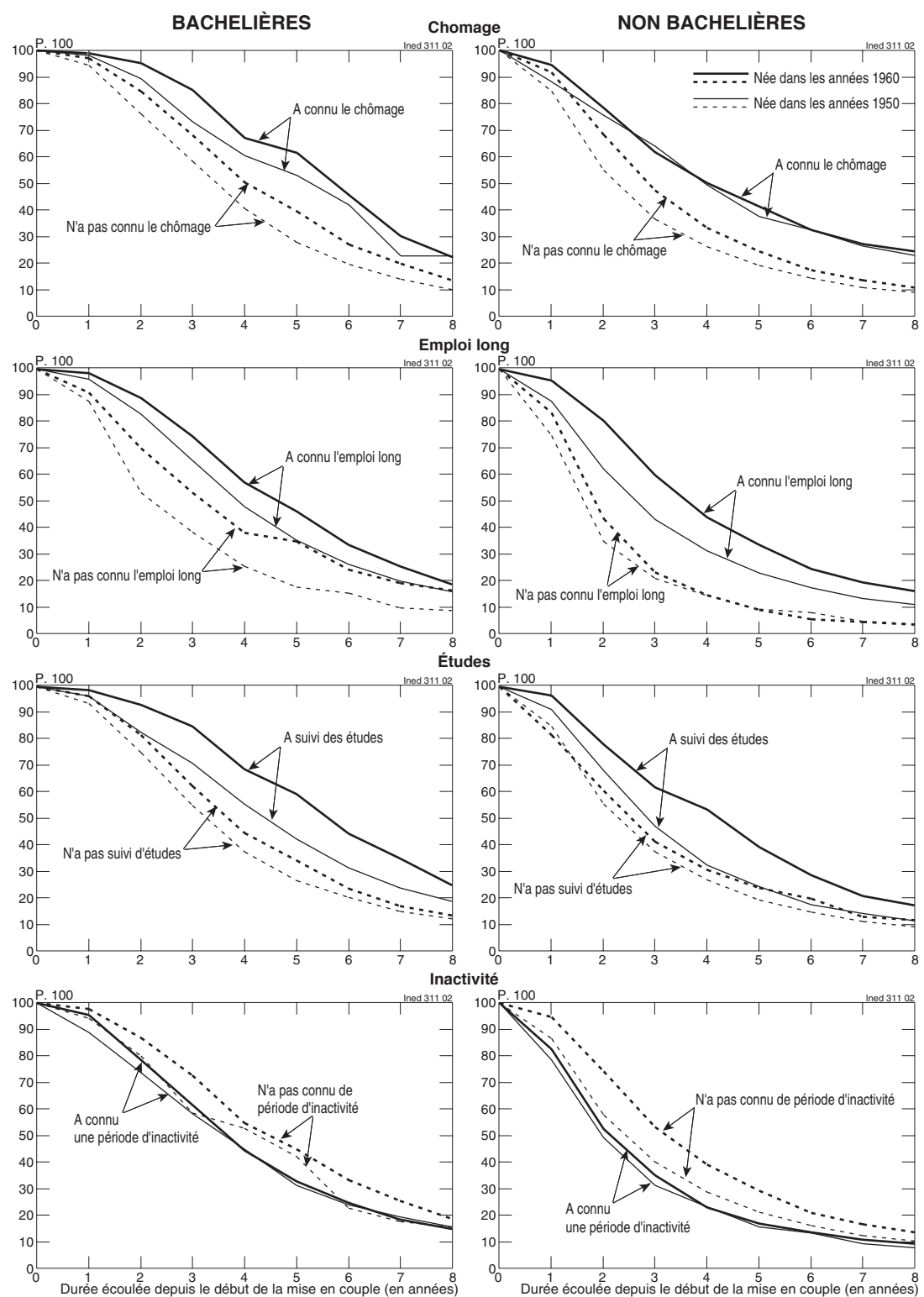

Figure 5.- Proportion de femmes sans enfant à chaque durée de vie en couple selon les situations vécues au regard de l'activité et le niveau de diplôme parmi les générations 1950 et 1960 (fonction de séjour)

Source: Insee, enquête Jeunes et carrières 1997. 


\section{... que celles qui ont eu des emplois stables ou même intermittents}

Parmi les femmes nées de 1952 à 1966 qui ont occupé au moins un emploi long, la moitié avaient un premier enfant au bout de 3,5 ans de vie en couple; cette durée médiane atteint 4,3 ans pour celles qui ont connu des périodes d'emplois courts ou intermittents, et même 4,5 ans parmi celles qui ont été confrontées au moins une fois à six mois de chômage continu. Ces résultats se déclinent selon la génération et le niveau de diplôme (tableau 1).

Comme on l'a déjà signalé, ces situations vis-à-vis de l'emploi ne sont pas exclusives; leurs effets peuvent donc être combinés. Pour mieux regarder l'impact du chômage (au sens de l'enquête, c'est-à-dire pendant au moins 6 mois), on mesure les effets exclusifs et croisés des situations «avoir connu l'emploi » (au sens large) et «avoir connu le chômage » à génération et niveau de diplôme donnés dans une analyse semiparamétrique à risques proportionnels (modèle de Cox). L'effet du chômage sur le retard du premier enfant est confirmé pour les femmes actives (tableau 3).

On peut cependant adresser une critique à ces résultats : plus le temps écoulé depuis la mise en couple est long, plus la femme a eu des chances de rencontrer chacune des situations recensées (emploi, chômage...). Il y a donc un biais possible à regarder des variables dont l'intensité est corrélée avec la durée observée. Pour répondre à cette objection, on peut faire intervenir l'événement «a rencontré le chômage » comme une variable dépendante du temps dans un modèle de Cox, c'est-àdire que l'événement n'intervient qu'à partir de l'année où il est signalé. Les résultats vont dans le sens des conclusions précédentes, mais le chômage continu, au sens de l'enquête, est un événement trop rare, quand on le considère année par année, à génération et niveau de diplôme fixés, pour donner seul des résultats significatifs aux seuils considérés auparavant.

Ces femmes ont rarement été confrontées à une période de chômage continu l'année même où elles se sont mises en couple (5\% de l'échantillon, tableau B, annexe, section 2). Ce constat est à relier au fait que les chômeuses temporisent déjà pour débuter une vie conjugale (Ekert et Solaz, 2000). Si l'on considère la situation d'activité l'année de la mise en couple, le chômage n'a pas un impact significativement différent de l'emploi continu. En revanche, si l'on distingue le fait d'avoir rencontré le chômage pour la première fois avant le début de la vie conjugale du fait de 1'avoir subi à partir de cette date, les résultats sont sans ambiguïté : le chômage retarde plus nettement l'arrivée du premier enfant lorsque les femmes ont connu cette expérience alors qu'elles étaient déjà en couple (tableau 4). 
TABLEAU 3.- DuRÉE ÉCOULÉE ENTRE LE DÉBUT DE LA VIE EN COUPLE ET LA NAISSANCE DU PREMIER ENFANT EN FONCTION DE DIFFÉRENTES SITUATIONS D'ACTIVITÉ VÉCUES DEPUIS LE DÉBUT DE LA VIE EN COUPLE (MODÈLES A ET B) (ANALYSES SEMI-PARAMÉTRIQUES) - ÉCHANTILLON DES FEMMES DE 31 À 45 ANS

\begin{tabular}{|c|c|c|c|c|}
\hline & \multicolumn{2}{|c|}{ Modèle A } & \multicolumn{2}{|c|}{ Modèle B } \\
\hline & Paramètre & $\begin{array}{c}\text { Effet } \\
\text { multiplicatif }\end{array}$ & Paramètre & $\begin{array}{c}\text { Effet } \\
\text { multiplicatif }\end{array}$ \\
\hline \multicolumn{5}{|l|}{ Génération } \\
\hline $1952-1954$ & $0,11^{*}$ & 1,12 & $0,11 * *$ & 1,12 \\
\hline $1955-1960$ & Réf. & 1,00 & Réf. & 1,00 \\
\hline 1961-1966 & $-0,12 * * *$ & 0,89 & $-0,12 * * *$ & 0,88 \\
\hline \multicolumn{5}{|l|}{ Niveau de diplôme } \\
\hline Supérieur à bac + 2 & $-0,55 * * *$ & 0,58 & $-0,50 * * *$ & 0,61 \\
\hline Bac à bac + 2 & $-0,22 * * *$ & 0,81 & $-0,22 * * *$ & 0,80 \\
\hline BEPC, CAP, BEP & Réf. & 1,00 & Réf. & 1,00 \\
\hline Sans diplôme ou CEP & $0,13 * * *$ & 1,14 & $0,19 * * *$ & 1,21 \\
\hline \multicolumn{5}{|l|}{ Situation d'activité(1) } \\
\hline Emploi et chômage & $-1,16^{* * *}$ & 0,32 & & \\
\hline Emploi sans chômage & $-0,55^{* * *}$ & 0,58 & & \\
\hline N'a pas occupé d'emploi & Réf. & 1,00 & & \\
\hline A connu le chômage & & & $-0,41 * * *$ & 0,67 \\
\hline N'a pas connu le chômage & & & Réf. & 1,00 \\
\hline A connu l'inactivité & & & $0,15 * * *$ & 1,16 \\
\hline N'a pas connu l'inactivité & & & Réf. & 1,00 \\
\hline \multicolumn{5}{|c|}{$\begin{array}{l}\text { * Significatif au seuil de } 5 \% \text {; ** significatif au seuil de } 5 \% \text {; } * * * \text { significatif au seuil de } 1 \% \text {. } \\
\text { (1) Périodes longues (au moins } 6 \text { mois) vécues à partir de l'année du début de la vie en couple et avant } \\
\text { d'avoir un enfant. } \\
\text { Lecture (modèle A) : toutes choses égales par ailleurs, une femme sans diplôme ou titulaire du CEP a } \\
1,14 \text { fois plus de chances d'avoir donné naissance à un premier enfant depuis le début de sa vie en couple } \\
\text { qu'une femme titulaire d'un diplôme de niveau BEPC, CAP ou BEP (référence). Au contraire, les chances } \\
\text { sont } 1,2 \text { fois plus faibles (multipliées par } 0,81 \text { ) pour les titulaires d'un diplôme de niveau baccalauréat ou } \\
\text { bac }+2 \text {. } \\
\text { Source } \text { : Insee, enquête Jeunes et carrières } 1997 \text {. }\end{array}$} \\
\hline
\end{tabular}

\section{Chômeuses et femmes au foyer n'ont pas les mêmes comportements familiaux}

En revanche, les femmes qui ont connu des périodes d'inactivité sont restées moins longtemps en couple sans enfant que les autres : la moitié d'entre elles avaient un enfant au bout de 2,3 ans de vie en couple (contre 3,2 ans pour celles qui n'ont pas été inactives). Encore une fois, ce résultat dépend de la génération et du niveau de diplôme (tableau 1).

L'impact de l'inactivité déclarée l'année de la mise en couple est très fort (tableau 4) : ce résultat semble indiquer que les femmes qui sont en dehors du marché du travail l'année où elles se mettent en couple ont, plus souvent que les autres, le projet d'avoir un enfant rapidement. Comme le suggérait Laurent Toulemon (dans Leridon, 1994, p. 174), «pour les femmes, la mise en couple avant toute activité professionnelle correspond 
plutôt à un choix centré sur la famille et les enfants ». A contrario, poursuivre des études alors qu'elles vivaient en couple a retardé l'arrivée du premier bébé des femmes de 31 à 45 ans. Ce résultat est plus ambigu lorsqu'il est décliné par génération et niveau de diplôme et que l'on veut comparer l'impact des études à celui de l'emploi ou du chômage. Les femmes nées dans les années 1950 qui ont poursuivi des études alors qu'elles vivaient en couple ont moins retardé la venue de leur premier enfant que les chômeuses; en revanche, les étudiantes nées dans les années 1960 sont encore plus dans une situation d'attente que les femmes actives de ces générations (tableau 1).

TABLEAU 4.- DuRÉE ÉCOULÉE ENTRE LE DÉBUT DE LA VIE EN COUPLE ET LA NAISSANCE DU PREMIER ENFANT EN FONCTION DE LA SITUATION D'ACTIVITÉ L'ANNÉE DU DÉBUT DE LA VIE EN COUPLE (MODËLE C) ET EN FONCTION DE LA CONFRONTATION AU CHÔMAGE AVANT OU APRÈS L'ANNÉE DU DÉBUT DE LA VIE EN COUPLE (MODÈLE D) (ANALYSES SEMI-PARAMÉTRIQUES) - ÉCHANTILLON DES FEMMES DE 31 À 45 ANS

\begin{tabular}{|c|c|c|c|c|}
\hline & \multicolumn{2}{|c|}{ Modèle C } & \multicolumn{2}{|c|}{ Modèle D } \\
\hline & Paramètre & $\begin{array}{c}\text { Effet } \\
\text { multiplicatif }\end{array}$ & Paramètre & $\begin{array}{c}\text { Effet } \\
\text { multiplicatif }\end{array}$ \\
\hline \multicolumn{5}{|l|}{ Génération } \\
\hline $1952-1954$ & $0,13 * *$ & 1,14 & $0,11^{*}$ & 1,12 \\
\hline $1955-1957$ & 0,02 & 1,02 & Réf. & 1,00 \\
\hline $1958-1960$ & Réf. & 1,00 & $0,01^{*}$ & 1,01 \\
\hline 1961-1963 & $-0,11 *$ & 0,90 & $-0,11 *$ & 0,89 \\
\hline 1964-1966 & $-0,13^{*}$ & 0,88 & $-0,13 *$ & 0,88 \\
\hline \multicolumn{5}{|l|}{ Niveau de diplôme } \\
\hline Supérieur à bac + 2 & $-0,41 * * *$ & 0,66 & $-0,48 * * *$ & 0,62 \\
\hline $\mathrm{Bac}+2$ & $-0,19 * * *$ & 0,82 & $-0,24 * * *$ & 0,79 \\
\hline $\mathrm{Bac}$ & $-0,18 * * *$ & 0,83 & $-0,18 * * *$ & 0,84 \\
\hline BEPC, CAP, BEP & Réf. & 1,00 & Réf. & 1,00 \\
\hline Sans diplôme ou CEP & $0,14 * * *$ & 1,15 & $0,21 * * *$ & 1,23 \\
\hline \multicolumn{5}{|l|}{$\begin{array}{l}\text { Situation d'activité l'année } \\
\text { de la mise en couple }\end{array}$} \\
\hline Inactive & $0,35 * * *$ & 1,41 & & \\
\hline Chômeuse & $-0,01$ & 0,92 & & \\
\hline Étudiante & $-0,12 * *$ & 0,88 & & \\
\hline Emplois courts & $-0,16 * *$ & 0,85 & & \\
\hline Emploi long & Réf. & 1,00 & & \\
\hline \multicolumn{5}{|l|}{ A connu le chômage } \\
\hline Avant la mise en couple & & & $-0,10^{*}$ & 0,90 \\
\hline Après la mise en couple & & & $-0,54 * * *$ & 0,58 \\
\hline N'a pas connu le chômage & & & Réf. & 1,00 \\
\hline \multicolumn{5}{|c|}{$\begin{array}{l}\text { * Significatif au seuil de } 5 \% \text {; ** significatif au seuil de } 5 \% \text {; } * * * \text { significatif au seuil de } 1 \% \text {. } \\
\text { Lecture (modèle C) : toutes choses égales par ailleurs, une femme sans diplôme ou titulaire du CEP a } \\
1,15 \text { fois plus de chances d'avoir eu un premier enfant depuis le début de sa vie en couple qu'une femme } \\
\text { qui a un diplôme de niveau BEPC, CAP ou BEP (référence). Au contraire, pour les bachelières, ces chances } \\
\text { sont près de } 1,2 \text { fois plus faibles (multipliées par } 0,83 \text { ). } \\
\text { Source : Insee, enquête Jeunes et carrières } 1997 \text {. }\end{array}$} \\
\hline
\end{tabular}


Les moins de 30 ans sur les traces de leurs aînées?

Quel est l'impact de la situation professionnelle sur la venue d'un premier enfant pour les femmes de 24 à 29 ans interrogées dans l'enquête Jeunes et carrières de 1997 ? Les calendriers professionnels des femmes de moins de 30 ans ont été recueillis suivant des modalités un peu plus détaillées que pour les plus âgées, mais ces légères différences ne remettent pas en cause la possibilité de comparaison avec les générations aînées (voir annexe, sections 1 et 2). Cette étude complémentaire porte sur 1565 femmes nées de 1968 à 1973 qui vivent ou ont vécu en couple; elles représentent environ 1700000 femmes de ces générations. Dans cet échantillon, les trajectoires «interrompues à droite» (770, soit 49,2\%) sont nettement plus fréquentes que dans le groupe précédent : plus jeunes, ces femmes sont plus nombreuses que leurs aînées à avoir vécu moins de 8 ans en couple à la date de l'enquête. Elles sont aussi plus souvent restées sans enfant dans la fenêtre observée, et se sont plus fréquemment séparées de leur premier conjoint (annexe, section 2, tableaux A et B). Pour ces jeunes générations, l'effectif étudié étant plus faible, il est nécessaire de regrouper les niveaux de diplômes et les générations : on distinguera les bachelières des non-bachelières, et les femmes nées de 1968 à 1970 de celles nées de 1971 à 1973 (figure 6).

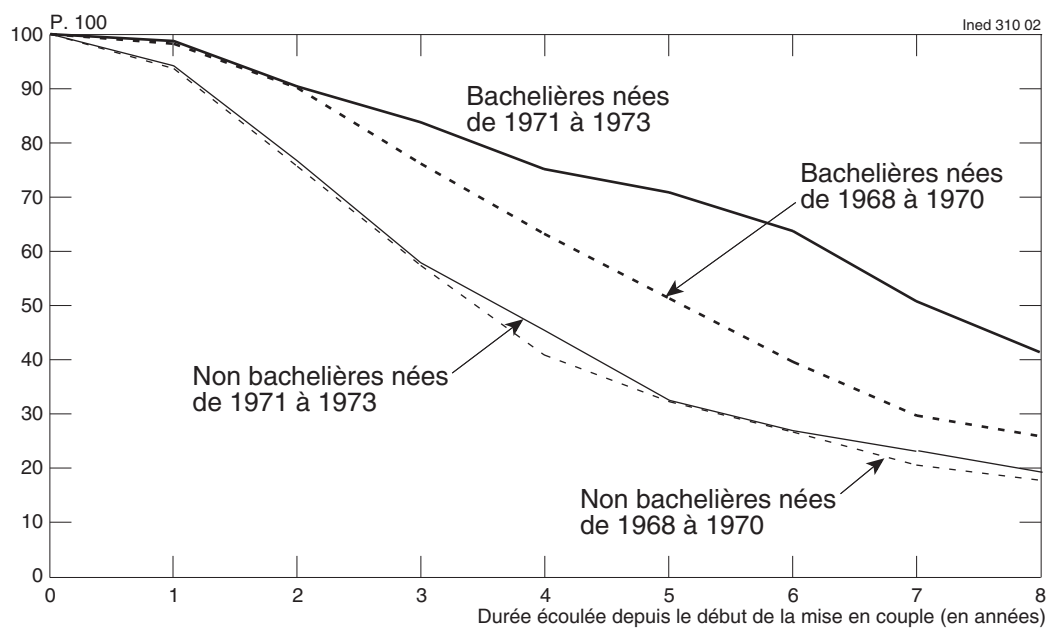

Figure 6.- Proportion de femmes sans enfant à chaque durée de vie en couple parmi les femmes les plus jeunes par génération et niveau de diplôme regroupés

Source: Insee, enquête Jeunes et carrières 1997. 
Une femme sur deux, née de 1968 à 1973, a attendu au moins 4,4 ans de vie conjugale pour avoir un premier enfant

Certaines tendances qui se dessinent chez les moins de 30 ans sont dans le prolongement de celles décrites pour les plus âgées. Ainsi, parmi les femmes de 24 à 29 ans (nées de 1968 à 1973), une sur deux a eu un premier enfant lors de ses 4,4 premières années de vie conjugale (au lieu de 3,0 ans pour les générations nées de 1952 à 1966), et les différences selon la génération subsistent : les femmes nées à la fin des années 1960 ont eu un premier enfant plus rapidement après le début de leur vie conjugale $(4,1$ ans) que celles nées au début des années 1970 (4,9 ans) (tableau A).

Le diplôme semble être très discriminant et le temps passé en couple sans enfant s'allongerait tout particulièrement d'une génération à la suivante lorsque la femme est diplômée. Cependant, poursuivre des études dissuade un certain nombre de jeunes femmes d'entamer une vie conjugale. À cet égard, l'observation est évidemment incomplète. De plus, à ces âges-là (24 à 29 ans au moment de l'enquête), un certain nombre de diplômées n'ont pas fini leurs études supérieures, ce qui peut être une explication au délai très important qui précède une première maternité chez les plus jeunes bachelières en couple.

Les moins de 30 ans ont, plus encore que leurs aînées, été touchées par la crise du marché du travail. Ainsi, ces jeunes femmes sont proportionnellement trois fois plus nombreuses à avoir connu le chômage ou des périodes d'emploi intermittent entre le début d'une première vie conjugale et l'année précédant l'arrivée du premier enfant ou la fin de l'observation (tableau B). Ces situations de précarité sur le marché du travail sont encore plus fréquentes chez les femmes peu ou pas diplômées et parmi les plus jeunes, nées de 1970 à 1973. Inversement, avoir occupé un emploi long est beaucoup moins répandu (47\% des femmes nées de 1968 à 1973 sur la période observée depuis la mise en couple, contre $77 \%$ pour les femmes nées de 1952 à 1966). Les femmes de moins de 30 ans sont également deux fois plus nombreuses en termes relatifs à poursuivre des études après le début de la vie en couple. En revanche, seulement une sur dix a connu l'inactivité sur la période observée et elles ne sont que $8 \%$ à se dire inactives l'année où elles se sont mises en couple alors que $16 \%$ ont été confrontées au chômage cette année-là (tableau B).

Dans les plus jeunes générations, le chômage a une influence plus marquée chez les moins diplômées, et comparable à celle de l'emploi intermittent

Comme pour leurs aînées, la durée médiane entre le début de la vie en couple et la naissance du premier enfant est nettement plus longue pour les femmes des jeunes générations qui poursuivent des études ou sont sur le marché du travail que pour les inactives. En effet, la moitié des jeunes femmes qui ont connu une période d'inactivité ont eu un premier enfant 
durant les 2,6 premières années de leur vie conjugale, alors que cette médiane est de deux années supplémentaires pour celles qui n'ont pas connu cette situation (tableau 1, figure 7).

Le temps passé en couple sans enfant est le plus court lorsque la femme est peu diplômée et qu'elle s'est retirée du marché du travail; l'effet de l'inactivité est particulièrement marqué pour celles qui étaient déjà femmes au foyer l'année du début de la vie conjugale (tableau 5, modèle f). Les autres situations d'activité l'année de la mise en couple ne ressortent pas significativement des modèles, bien que la fréquence du chômage, des études ou des emplois courts soit plus importante pour les jeunes femmes de cet échantillon que pour leurs aînées.

TABleau 5.- DuRÉE ÉCOUlÉE ENTRE LE DÉBUT DE LA VIE EN COUPLE ET LA NAISSANCE DU PREMIER ENFANT EN FONCTION DES SITUATIONS D'ACTIVITÉ RENCONTRÉES DEPUIS LE DÉBUT DE LA VIE EN COUPLE (MODĖLE E) ET EN FONCTION DE LA SITUATION D’ACTIVITÉ VÉCUE L’ANNÉE DU DÉBUT DE LA VIE EN COUPLE (MODÈLE F) (ANALYSES SEMI-PARAMÉTRIQUES) - ÉCHANTILLON DES FEMMES DE 24 À 29 ANS

\begin{tabular}{|c|c|c|c|c|}
\hline & \multicolumn{2}{|c|}{ Modèle E } & \multicolumn{2}{|c|}{ Modèle F } \\
\hline & Paramètre & $\begin{array}{c}\text { Effet } \\
\text { multiplicatif }\end{array}$ & Paramètre & $\begin{array}{c}\text { Effet } \\
\text { multiplicatif }\end{array}$ \\
\hline \multicolumn{5}{|l|}{ Génération } \\
\hline $1968-1970$ & Réf. & 1,00 & Réf. & 1,00 \\
\hline $1971-1973$ & $-0,19 *$ & 0,83 & $-0,23 * *$ & 0,80 \\
\hline \multicolumn{5}{|l|}{ Diplôme } \\
\hline Supérieur à bac + 2 & $-1,30 * * *$ & 0,27 & $-1,23 * * *$ & 0,29 \\
\hline Bac, bac +2 & $-0,46 * * *$ & 0,63 & $-0,42 * * *$ & 0,65 \\
\hline BEPC, CAP, BEP & Réf. & 1,00 & Réf. & 1,00 \\
\hline Sans diplôme ou CEP & $0,22 *$ & 1,25 & 0,15 & 1,16 \\
\hline \multicolumn{5}{|l|}{$\begin{array}{l}\text { Situation d'activité l'année } \\
\text { de la mise en couple }\end{array}$} \\
\hline Inactive & & & $0,79 * * *$ & 2,19 \\
\hline Chômeuse & & & 0,02 & 1,02 \\
\hline Étudiante & & & Réf. & 1,00 \\
\hline Emplois courts & & & $-0,07$ & 0,93 \\
\hline Emploi long & & & $-0,04$ & 0,96 \\
\hline \multicolumn{5}{|l|}{ Activité } \\
\hline A connu le chômage & $-0,45 * * *$ & 0,64 & & \\
\hline N'a pas connu le chômage & Réf. & 1,00 & & \\
\hline A connu l'inactivité & $0,46 * * *$ & 1,59 & & \\
\hline N'a pas connu l'inactivité & Réf. & 1,00 & & \\
\hline \multicolumn{5}{|c|}{$\begin{array}{l}\text { * Significatif au seuil de } 5 \% \text {; } * * \text { significatif au seuil de } 5 \% \text {; } * * * \text { significatif au seuil de } 1 \% \text {. } \\
\text { Lecture (modèle E) : toutes choses égales par ailleurs, une femme sans diplôme ou titulaire du CEP a } \\
1,25 \text { fois plus de chances d'avoir eu un premier enfant depuis le début de sa vie en couple qu'une femme } \\
\text { titulaire d'un diplôme de niveau BEPC, CAP ou BEP (référence). Au contraire, pour les titulaires d'un } \\
\text { diplôme de niveau baccalauréat ou bac + } 2 \text {, ces chances sont } 1,6 \text { fois plus faibles (multipliées par } 0,63 \text { ). } \\
\text { Source : Insee, enquête Jeunes et carrières } 1997 \text {. }\end{array}$} \\
\hline
\end{tabular}




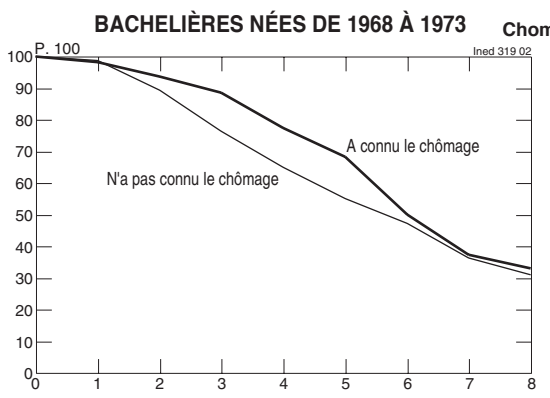

NON BACHELIÈRES NÉES DE 1968 À 1973
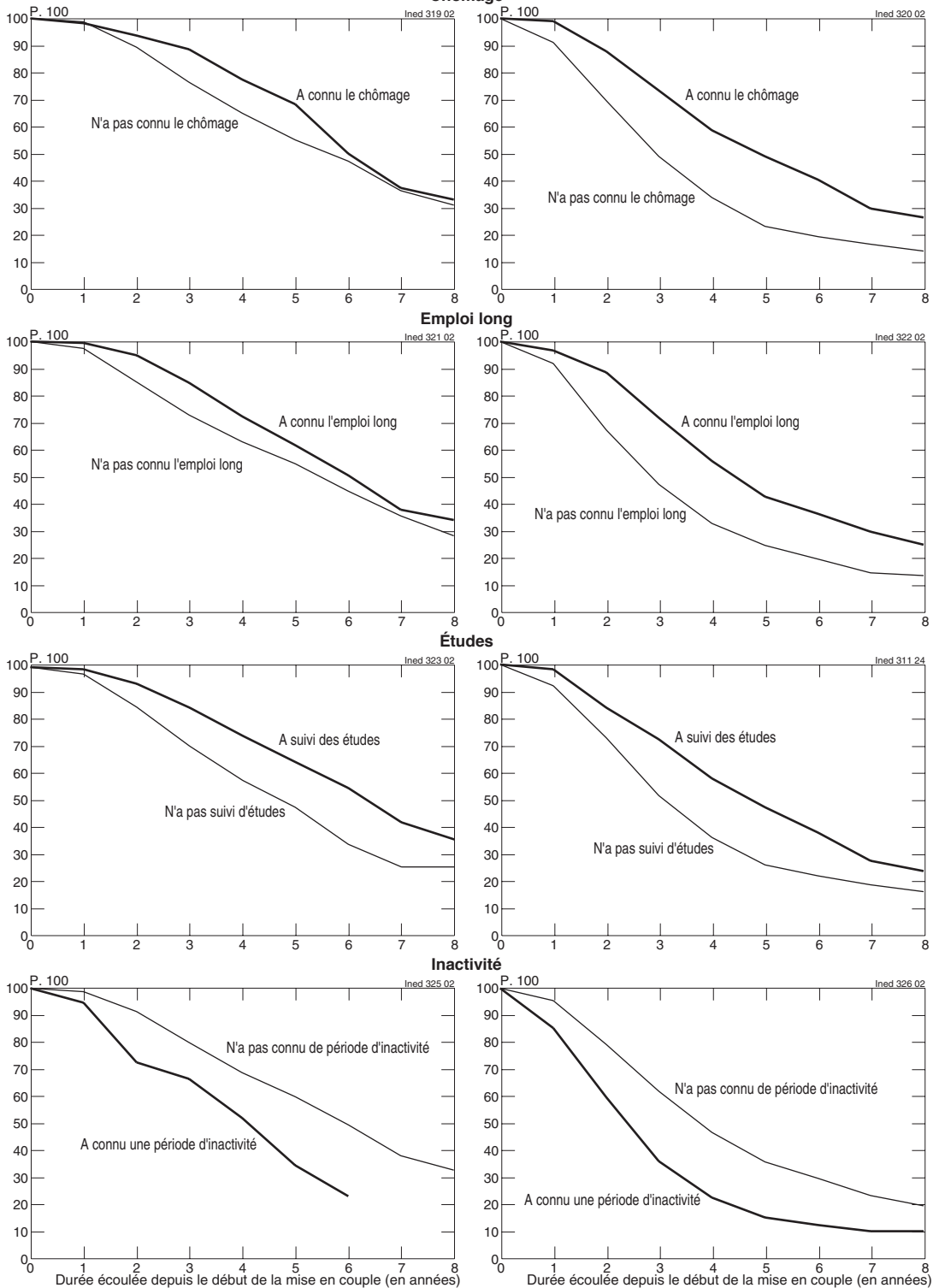

Figure 7.- Proportion de femmes sans enfant à chaque durée de vie en couple selon les situations vécues au regard de l'activité et le niveau de diplôme parmi les femmes les plus jeunes (fonction de séjour) 
Comme pour l'échantillon précédent, on a testé l'influence des différentes situations d'activité (chômage, emploi long, emplois courts, études) sur la durée écoulée entre le début de la vie en couple et l'année précédant la naissance du premier enfant. Il ressort alors nettement que chacune de ces situations allonge très sensiblement le temps passé en couple sans enfant, à diplômes et générations donnés, contrairement à l'inactivité. Les plus diplômées restent toujours plus longtemps en couple sans enfant, quelle que soit la situation professionnelle rencontrée.

L'influence du chômage est très nette chez les moins diplômées (tableau 1). Ainsi, les femmes non bachelières ayant connu le chômage depuis leur mise en couple retardent la venue du premier enfant de près de 2 années (en durée médiane) par rapport à celles qui n'ont pas vécu cette situation : 4,9 ans contre 3 ans.

L'effet retard de l'emploi long est également plus fort pour les nonbachelières que pour les bachelières. Quant aux périodes d'emploi intermittent, loin d'avoir un effet intermédiaire entre emploi long et chômage comme pour les femmes nées de 1952 à 1966, elles retardent autant que le chômage l'arrivée du premier enfant parmi les jeunes générations nées de 1968 à 1973 : la durée médiane écoulée avant une première naissance atteint 5,5 ans (tableau 1).

\section{Conclusion}

La méthode d'analyse biographique appliquée à l'enquête Jeunes et carrières permet donc d'éclairer la question de l'impact du chômage sur la vie familiale. On peut affirmer que le chômage retarde la venue d'un premier enfant lorsqu'il touche les jeunes femmes qui vivent en couple. Ainsi, la confrontation avec une période de chômage a incité celles qui sont nées entre 1952 et 1973 à reporter la venue d'un premier enfant en attendant des jours meilleurs. On a montré que cette tendance est encore plus accusée lorsque la femme est peu diplômée et appartient aux générations les plus récentes.

Une différence essentielle oppose la situation de femme au foyer à toutes les autres (emploi, chômage ou poursuite d'études). L'inactivité, surtout au début de la vie conjugale, correspond à des premières maternités beaucoup plus rapides, qui reflètent sans doute des projets d'enfant avancés. Ce résultat confirme qu'une période de chômage n'est pas assimilable à une période d'inactivité : au regard de la fécondité, les chômeuses ne sont pas des femmes au foyer.

Des plus âgées aux plus jeunes, on observe une forte continuité dans les comportements, malgré un contexte économique et social qui a changé. La diffusion des diplômes a renforcé l'écart entre celles qui ont interrompu leur scolarité sans résultat et celles qui ont suivi des études supérieures; la banalisation du chômage auquel s'ajoutent, pour les plus 
jeunes, les périodes d'emploi intermittent, ont fait évoluer les comportements : selon le niveau de diplôme, on voit apparaître des groupes pour lesquels ces périodes d'incertitude sur le marché du travail correspondent à des réalités bien différentes. Ainsi, le chômage des moins diplômées, plus crucial sans doute dans leur revenu familial, a un impact plus fort sur leur fécondité. Quant aux emplois intermittents, leur influence se rapproche de celle du chômage chez les plus jeunes.

Les projets d'enfants sont donc aussi réfléchis en fonction de paramètres économiques et dépendent de l'évolution des situations professionnelles. Les femmes qui sont dans une situation précaire sur le marché du travail retardent la venue du premier enfant tant que leur projet d'emploi stable ou de fin d'études n'est pas réalisé. Ces résultats rappellent, si besoin était, que le travail des femmes correspond à des attentes en termes de revenus et de reconnaissance sociale, peut-être aussi de métier ou de carrière. Par rapport aux décisions d'ordre familial, ces apports ne sont pas ressentis que comme un appoint, et ils interfèrent avec les priorités des couples.

Dans une société où la contraception permet le plus souvent de mâ̂triser le moment de devenir mère et où l'activité professionnelle des femmes est progressivement devenue la norme, les projets et calendriers familiaux tiennent compte de la situation professionnelle vécue et anticipée par les femmes. Toute analyse introduisant de manière symétrique et détaillée la vie familiale, professionnelle et migratoire des femmes et de leurs conjoints permettra d'avancer dans la compréhension des comportements.

\section{ANNEXE}

\section{L'enquête Jeunes et carrières}

L'enquête Jeunes et carrières est une enquête complémentaire à l'enquête Emploi réalisée en vis-à-vis par les enquêteurs de l'Insee en mars 1997. La collecte, en grande partie assistée par ordinateur (méthode CAPI, sauf pour le calendrier), a concerné les personnes de 19 à 45 ans du tiers sortant de l'enquête Emploi, soit un échantillon représentatif de la population constitué de 20770 individus dont 12400 personnes de 30 à 45 ans. Les calendriers rétrospectifs relèvent, depuis l'année des 16 ans, les événements familiaux (mise en couple, mariage, séparation, naissance des enfants), les changements de résidence et les périodes d'études et d'activité vécus par la personne. L'appel à la mémoire est facilité par la juxtaposition de ces différents types d'événements.

\section{Description de l'échantillon}

On a choisi d'étudier ici la population des femmes la plus soumise au risque d'avoir un enfant, et on prend comme origine le début de la vie en couple $\left(\mathrm{t}_{0}\right)$. En effet, partir de l'âge de 16 ou 18 ans reviendrait à mélanger les effets de retard à la mise en couple et ceux relatifs à la date de naissance du premier enfant. Or, ce sont 
les situations d'activité postérieures au début de la vie en couple qui sont les plus influentes. Pour des raisons similaires, on a également évité de prendre comme point de départ la fin des études : beaucoup de femmes débutent une vie de couple avant d'avoir fini leurs études.

On analyse donc la durée écoulée entre le début de la vie conjugale $\left(\mathrm{t}_{0}=\right.$ première date de mise en couple $)$ et la date de naissance du premier enfant $\left(\mathrm{t}_{1}\right)$, si cette naissance survient dans les 8 ans suivant la première mise en couple sans qu'il y ait eu séparation des conjoints. Au-delà de 8 ans après la mise en couple, le modèle à risques proportionnels que nous utilisons n'est plus applicable. Ces situations, relativement rares (moins de $5 \%$ ), ne peuvent être étudiées de la même façon.

Font partie de l'analyse comme «données interrompues à droite »: les femmes qui restent avec leur premier conjoint mais n'ont pas eu d'enfant durant les 8 premières années de vie conjugale $\left(\mathrm{t}_{1}=\mathrm{t}_{0}+8\right)$, celles qui se séparent de ce conjoint avant d'avoir un enfant et avant 8 ans de vie commune $\left(\mathrm{t}_{1}=\right.$ date de séparation), celles pour qui la date d'enquête intervient avant 8 ans de vie commune et avant qu'elles aient eu un enfant $\left(t_{1}=1997\right)$.

En ce qui concerne l'activité, plusieurs situations (emploi, chômage, poursuite d'études, inactivité) peuvent être mentionnées pour une même année dans le calendrier de l'enquête. Toutes sont prises en compte sur la période étudiée. Pour les jeunes, les situations professionnelles sont plus détaillées.

\section{a) Les femmes de 31 à 45 ans}

Sur 6256 femmes nées de 1952 à 1966 présentes dans l'échantillon, 5771 ont vécu en couple; on élimine ainsi environ 12,4\% des plus jeunes (nées de 1964 à 1966) et 5,1\% des plus âgées (nées de 1952 à 1954).

Parmi les femmes ayant vécu en couple, 163 sont éliminées car elles ont eu un enfant avant la première mise en couple et 102 autres le sont car aucune situation d'activité n'est remplie dans le calendrier à la date de mise en couple, ce qui ne permet pas de les situer par rapport aux études et au marché du travail.

Le fichier final est donc constitué de 5506 femmes (tableau A); 4629 femmes ont eu un enfant moins de 9 ans après le début de leur première vie en couple et sans être séparées de leur premier conjoint. 503 (9,1\%) n'ont pas eu d'enfant, 283 en ont eu un plus de 8 ans après le début de leur première vie en couple, 302 se sont séparées de leur conjoint avant 8 ans de vie commune, dont 91 avant d'avoir eu leur premier enfant. Au total, 877 femmes (soit 15,9\%) sont incluses dans l'analyse comme données dites « censurées à droite ».

En ce qui concerne la situation au regard de l'activité, les calendriers annuels ne signalent que des périodes longues (au moins 6 mois). On distingue :

- le chômage continu : «au chômage avec recherche d'emploi, de manière continue »;

- l'emploi long, continu : « en emplois de plus de 6 mois (recueillis sur deux lignes selon les changements d'employeurs) »;

- l'emploi intermittent : « en emplois courts alternant avec des périodes sans emploi ou périodes d'apprentissage »;

- l'inactivité : « inactif, femme au foyer»;

- en études: «périodes d'études ou en formation continue ou reprise d'études ». 
L'année où elles ont débuté une vie en couple, deux femmes sur trois ont occupé un emploi de longue durée (66\%), moins d'une sur cinq poursuivait des études $(17,9 \%)$, tandis que la proportion d'inactives $(13,2 \%)$ est proche de celles qui ont eu des emplois intermittents $(9,3 \%)$; enfin, rares sont celles qui ont connu, cette année-là, une période de chômage continu (5,3\%) (tableau B).

\section{TABLEAU A. - STRUCTURE DES ÉCHANTILLONS DE L'ENQUÊTE JEUNES ET CARRIÈRES ET DURÉE ÉCOULÉE ENTRE LE DÉBUT DE LA VIE EN COUPLE ET LA NAISSANCE DU PREMIER ENFANT (DURÉE MÉDIANE EN ANNÉES)}

\begin{tabular}{|c|c|c|c|c|c|}
\hline \multirow[b]{2}{*}{ Génération } & \multirow{2}{*}{$\begin{array}{l}\text { Nombre de } \\
\text { femmes }\end{array}$} & \multirow{2}{*}{$\begin{array}{c}\% \text { dans l'échan- } \\
\text { tillon }\end{array}$} & \multirow[b]{2}{*}{ \% pondéré } & \multicolumn{2}{|c|}{ Durée médiane en années sur } \\
\hline & & & & Effectifs bruts & $\begin{array}{l}\text { Effectifs } \\
\text { pondérés }\end{array}$ \\
\hline \multicolumn{6}{|c|}{ Échantillon des femmes de 31 à 45 ans } \\
\hline $1952-1954$ & 1030 & 18,7 & 20,0 & 2,43 & 2,45 \\
\hline $1955-1957$ & 1151 & 20,9 & 20,3 & 2,82 & 2,88 \\
\hline $1958-1960$ & 1114 & 20,2 & 20,5 & 3,02 & 3,10 \\
\hline $1961-1963$ & 1174 & 21,3 & 20,1 & 3,35 & 3,39 \\
\hline 1964-1966 & 1037 & 18,8 & 19,1 & 3,66 & 3,72 \\
\hline Total 1952-1966 & 5506 & 100,0 & $\begin{array}{c}100,0 \\
(n=5660000)\end{array}$ & 3,02 & 3,08 \\
\hline \multicolumn{6}{|c|}{ Échantillon des femmes de 24 à 29 ans } \\
\hline $1968-1970$ & 873 & 55,8 & 56,8 & 4,10 & 4,16 \\
\hline 1971-1973 & 692 & 44,2 & $\begin{array}{c}43,2 \\
100,0\end{array}$ & 4,90 & 5,00 \\
\hline Total 1968-1973 & 1565 & 100,0 & $(n=1694600)$ & 4,40 & 4,44 \\
\hline
\end{tabular}

Avant d'avoir un enfant, les types de situations rencontrées par les jeunes femmes en couple se multiplient : plus des trois quarts ont eu au moins une période d'emploi long; ce pourcentage est proche de $84 \%$ parmi les plus diplômées. Les périodes d'emploi intermittent et de chômage sont surtout le lot des plus jeunes et des moins diplômées. Le chômage continu touche environ une femme sur dix. Parmi les plus diplômées, plus d'une femme sur trois poursuit ses études après le début de sa vie en couple. Parmi celles qui n'ont pas le baccalauréat, une sur cinq connaît au moins une période d'inactivité (tableau B).

La probabilité d'avoir connu ces différentes situations n'est pas indépendante des caractéristiques des jeunes femmes. Les méthodes utilisées permettent de séparer les effets dus à chacune des variables prises en compte dans le modèle.

\section{b) Les femmes de 24 à 29 ans}

L'échantillon « jeunes » est constitué de 1565 jeunes femmes nées de 1968 à 1973. Parmi elles, 737 (47,1\%) n'ont pas eu d'enfant, 8 en ont eu un plus de 8 ans après le début de leur première vie en couple, 141 se sont séparées de leur conjoint avant 8 ans de vie commune dont 25 avant d'avoir eu leur premier enfant. Au total, 795 femmes ont eu un enfant moins de 9 ans après le début de leur première vie en couple et sans être séparées de leur premier conjoint. Les autres (770, soit 49,2\%) sont incluses dans l'analyse comme données dites « censurées à droite». 
TABLEAU B. - SITUATIONS D’ACTIVITÉ RENCONTRÉES ${ }^{(1)}$ À PARTIR DU DÉBUT DE LA VIE EN COUPLE ET AVANT LA NAISSANCE DU PREMIER ENFANT

\begin{tabular}{|c|c|c|c|c|c|c|c|}
\hline & \multicolumn{2}{|c|}{ Niveau de diplôme (\%) } & \multicolumn{2}{|c|}{ Générations (\%) } & \multicolumn{2}{|c|}{ Ensemble } & $\begin{array}{l}\text { Situation } \\
\text { en t }_{0}^{(2)}\end{array}$ \\
\hline \multicolumn{8}{|c|}{ Échantillon des femmes de 31 à 45 ans } \\
\hline & $\begin{array}{l}\text { Bac ou } \\
\text { plus }\end{array}$ & $\begin{array}{c}\text { Inférieur } \\
\text { au bac }\end{array}$ & $1952-1960$ & $1961-1966$ & $\%$ & Effectif & $\%$ \\
\hline Emploi long & 83,6 & 74,2 & 78,8 & 75,4 & 77,5 & 4265 & 66,0 \\
\hline Emplois courts & 12,4 & 13,5 & 9,6 & 18,5 & 13,1 & 723 & 9,3 \\
\hline Chômage & 7,8 & 10,1 & 6,4 & 13,6 & 9,3 & 512 & 5,3 \\
\hline Études & 38,0 & 9,8 & 17,5 & 22,8 & 19,6 & 1080 & 17,9 \\
\hline Inactivité & 10,2 & 21,0 & 17,7 & 16,5 & 17,2 & 947 & 13,2 \\
\hline Ensemble (effectif) & 1916 & 3590 & 3295 & 2211 & 100,0 & 5506 & 5506 \\
\hline \multicolumn{8}{|c|}{ Échantillon des femmes de 24 à 29 ans } \\
\hline & $\begin{array}{c}\text { Bac ou } \\
\text { plus }\end{array}$ & $\begin{array}{l}\text { Inférieur } \\
\text { au bac }\end{array}$ & $1968-1970$ & $1971-1973$ & $\%$ & Effectif & $\%$ \\
\hline Emploi long & 53,0 & 41,0 & 54,9 & 36,7 & 46,8 & 733 & 34,0 \\
\hline Emplois courts & 36,7 & 40,9 & 35,3 & 43,4 & 38,8 & 608 & 26,4 \\
\hline Chômage & 21,4 & 34,9 & 25,8 & 31,6 & 28,4 & 444 & 16,1 \\
\hline Études & 56,7 & 28,4 & 38,5 & 46,8 & 42,2 & 660 & 37,6 \\
\hline Inactivité & 4,9 & 16,1 & 11,8 & 9,2 & 10,7 & 167 & 7,8 \\
\hline Ensemble (effectif) & 760 & 805 & 873 & 692 & 100,0 & 1565 & 1565 \\
\hline \multicolumn{8}{|c|}{$\begin{array}{l}\text { (1) Au sens de l'enquête (périodes continues d'au moins } 6 \text { mois pour emploi long, chômage, inactivité). Plusieurs } \\
\text { situations peuvent avoir été rencontrées une même année. } \\
\text { (2) } \mathrm{t}_{0}=\text { année du début de la première vie en couple. } \\
\text { Source : Insee, enquête Jeunes et carrières } 1997 \text {. }\end{array}$} \\
\hline
\end{tabular}

Le calendrier utilisé pour retracer les situations professionnelles des jeunes diffère de celui des femmes appartenant aux générations plus anciennes, afin de mieux prendre en compte leur insertion et de rester cohérent avec les enquêtes précédentes. Dans le cas où la jeune enquêtée a connu plusieurs situations différentes la même année, on trouve, outre la situation principale de l'année, une situation « secondaire » (signalée si elle a duré au moins trois mois ou si elle a coexisté avec la situation principale).

Les regroupements de situations professionnelles des jeunes sont réalisés de façon à rester cohérents par rapport à ceux des plus âgés :

- le chômage: situation principale ou secondaire «au chômage avec recherche d'emploi »;

- l'emploi long : situation principale ou secondaire «salarié pour une durée non limitée à temps complet ou aide familial ou indépendant »;

- l'emploi court ou intermittent: situation principale ou secondaire « en apprentissage ou contrats à durée déterminée, mission d'intérim, travail saisonnier »;

- l'inactivité : situation principale ou secondaire « inactif, femme au foyer »;

- les études : situation principale ou secondaire «élève, étudiant, stagiaire non rémunéré ou stagiaire de la formation professionnelle, contrat d'aide à l'emploi ou élève ou étudiant rémunéré ». 
L'année où elles ont commencé à vivre en couple, $34 \%$ seulement des jeunes femmes de l'échantillon avaient un emploi long, plus d'un tiers poursuivaient des études $(38 \%)$, la proportion d'inactives $(7,8 \%)$ est très inférieure à celle des femmes qui occupaient des emplois courts $(26,4 \%)$ et $16,1 \%$ ont connu, cette année-là, une période de chômage, soit beaucoup plus que les générations précédentes (tableau B).

Avant d'avoir un enfant, les types de situations rencontrées par les jeunes femmes en couple se multiplient : près de la moitié $(46,8 \%)$ ont eu au moins une période d'emploi long; ce pourcentage est proche de $53 \%$ parmi les plus diplômées. Les périodes d'emploi court et de chômage sont surtout le lot des plus jeunes et des moins diplômées. Le chômage touche près de 3 femmes sur 10. Parmi les plus diplômées, 56,7 \% poursuivent des études après le début de leur vie en couple ; parmi celles qui n'ont pas le baccalauréat, $16 \%$ connaissent au moins une période d'inactivité (tableau B).

\section{c) Pondérer ou non?}

L'échantillon est représentatif de la population totale et les pondérations tiennent compte des taux de non-réponse (relativement faibles : autour de $7 \%$ ) par sexe, âge, type d'activité et taille d'unité urbaine. Ainsi, en toute rigueur, les 5506 femmes de l'échantillon représentent environ 5660000 femmes de 31 à 45 ans en mars 1997 et l'échantillon «jeunes » constitué de 1565 jeunes femmes nées de 1968 à 1973 représente 1695000 femmes (tableau A). Faut-il ou non pondérer les effectifs dans les analyses? Dans une analyse transversale, chaque individu enquêté représente clairement un poids dans la base de sondage. Dans les analyses longitudinales, le débat n'est pas clos. Comme l'échantillon est représentatif au moment de l'enquête, on peut supposer qu'il l'est aussi du passé des individus représentés; mais cela est déjà plus discutable (Hoem, 1985), la condition pour pouvoir utiliser un échantillon non pondéré étant que celui-ci soit non informatif. Malheureusement, cette condition n'est jamais parfaitement vérifiée. Il est toutefois indispensable de travailler aussi sur des effectifs non pondérés pour s'assurer de la significativité des résultats.

En négligeant les nouvelles non-réponses induites par les calendriers « mal remplis », on a recalculé un certain nombre de résultats en attribuant à chaque individu son poids dans l'enquête. Les résultats pondérés diffèrent peu des résultats non pondérés, témoignant de la qualité du sondage de départ (tableau A).

\section{Ce que l'analyse ne dit pas}

Le modèle présenté dans cet article permet d'avancer dans l'analyse des interactions entre marché du travail et comportements familiaux, sans pour autant prendre en compte la totalité des facteurs explicatifs. Sans remettre en cause les conclusions présentées ici, on aimerait affiner l'explication.

\section{a) Problèmes de couples...}

Tout d'abord, on sait que les trajectoires des conjoints ne sont pas indépendantes : au vu de la migration, du type d'emploi, etc., l'une dépend en partie de l'autre et certaines caractéristiques du couple, comme la différence d'âge entre les conjoints, ont une influence significative sur leurs parcours professionnels (Courgeau et Meron, 1996). A fortiori, l'enfant est un projet de couple et la situation d'activité du futur père interagit sûrement avec celle de la mère. Cet aspect ne peut pas être pris en compte ici car l'enquête ne porte que sur les conjoints présents 
en mars 1997 et rien n'est dit sur les premiers conjoints lorsqu'il y a eu séparation. Or, l'approche biographique nécessite que l'on fasse intervenir ces caractéristiques au moment où elles sont présentes. Pour pallier cet inconvénient dû aux séparations (au demeurant d'autant plus nombreuses qu'il s'agit de générations récentes), on aurait pu étudier uniquement la venue du premier enfant des femmes restées avec le même conjoint depuis la première mise en couple, mais cela aurait introduit des biais de sélection tout à fait dommageables. Ces problèmes se posent également lorsque l'on souhaite tenir compte des lieux de résidence successifs de l'individu. Enfin, il serait intéressant de savoir si l'influence du chômage de la femme est ou non tempérée par la stabilité de l'emploi de son conjoint, si le chômage de l'homme recule moins, autant ou plus le projet d'enfant que celui de la femme, ou encore de mesurer l'impact du chômage cumulé par les deux conjoints ou l'influence de certains types d'emplois.

\section{b) ...et de santé}

Enfin, toute donnée relative à la santé ou à d'éventuels problèmes de contraception ou d'infertilité étant absente du fichier de départ, cette analyse ne peut les prendre en compte, ce qui revient à considérer que ces problèmes sont indépendants des variables explicatives, donc, pour l'essentiel, de la génération, du niveau de diplôme et de la situation d'activité des femmes. Or, on sait que certains problèmes de santé sont plus fréquents chez les chômeurs que chez les autres actifs. Certains inactifs le sont pour raison de santé, etc. Il paraît cependant peu vraisemblable que de tels problèmes expliquent l'ampleur des différences constatées.

Par ailleurs, si l'infertilité est liée à l'âge, elle contribue peut-être à allonger la période sans enfant des femmes les plus diplômées et des plus jeunes qui se mettent en couple plus tard. Cette information est en tout état de cause suffisamment ténue pour être contenue dans l'information déjà expliquée puisque l'âge à la mise en couple, une fois pris en compte la génération et le niveau de diplôme, n'apparaît pas comme variable complémentaire.

\section{c) Quelques pistes pour l'avenir}

L'approche biographique, bien adaptée à ces problématiques, pourrait permettre d'explorer d'autres questions. Il serait intéressant d'analyser les autres intervalles génésiques pour voir si l'effet du chômage sur le retard de la venue du premier enfant se confirme sur les rangs de naissances plus élevés. On pourrait aussi étudier les parcours des hommes et comparer leurs arbitrages à ceux des femmes...

Les limites de l'enquête Jeunes et carrières invitent à rêver d'autres sources de données, où les calendriers professionnel, familial et migratoire seraient plus complets. Une autre piste de recherche intéressante pourrait s'ouvrir en confrontant point de vue de l'individu et stratégie de l'entreprise. L'introduction de caractéristiques de l'employeur dans l'analyse des comportements individuels, l'étude simultanée de la biographie des personnes et de l'histoire des entreprises pour lesquelles elles travaillent permettraient de cerner de façon plus fine comment se construisent certains comportements familiaux. 


\section{RÉFÉRENCES}

Aerts Anne-Thérèse, Mercier Marie-Annick, 2001, «Enquête sur l'emploi de mars 2001 : la forte hausse de l'emploi se poursuit », Insee Première, $\mathrm{n}^{\circ} 785,5 \mathrm{p}$.

BARrère-Maurisson Marie-Agnès, Marchand Olivier, 2000, «Famille et marché du travail dans les pays développés au seuil de l'an 2000 », Premières informations et premières synthèses, $\mathrm{n}^{\circ} 11.1,8 \mathrm{p}$.

Baudelot Christian, Establet Roger, 2000, Avoir 30 ans en 1968 et en 1998, Éditions du Seuil, $217 \mathrm{p}$.

Bonnet Carole, LabBÉ Morgane, 1999, «L'activité professionnelle des femmes après la naissance de leurs deux premiers enfants : l'impact de l'allocation parentale d'éducation », Études et résultats, $\mathrm{n}^{\circ} 37,7 \mathrm{p}$.

BRunet François, Minni Claude, 2000, «L'activité des 15-29 ans : stabilisation depuis 1995 », Premières informations et premières synthèses, $\mathrm{n}^{\circ} 08.3,11 \mathrm{p}$.

Courgeau Daniel, Lelièvre Éva, 1989, Analyse démographique des biographies, Paris, Ined, $269 \mathrm{p}$.

Courgeau Daniel, Meron Monique, 1996, «Mobilité résidentielle, activité et vie familiale des couples », Économie et statistique, ${ }^{\circ} 290$.

Demazière Didier, Dubar Claude, 1997, Analyser les entretiens biographiques : l'exemple des récits d'insertion, Essais et Recherches, Nathan, 350 p.

Desplanques Guy, Saboulin Michel de, 1986, «Mariage et premier enfant : un lien qui se défait », Économie et Statistique, n 187 , p. 31-45.

Desplanques Guy, 1996, «Un facteur de naissances tardives : le diplôme », Population, l'état des connaissances : la France, l'Europe, le Monde, Ined/La découverte, p. 17.

Dormont Brigitte, Dufour-KiPPElEn Sandrine, 2000, «Insertion professionnelle et autonomie résidentielle : le cas des jeunes peu diplômés », Économie et statistique, $\mathrm{n}^{\circ}$ 337-338.

EKert-JafFe Olivia, Solaz Anne, 2000, Unemployment and family formation in France, Ined, (coll. Dossiers et Recherches, $\mathrm{n}^{\circ} 82$ ), $26 \mathrm{p}$.

Estrade Marc-Antoine, Minni Claude, 1996, «La hausse du niveau de formation », Insee Première, $\mathrm{n}^{\circ}$ 488, 4 p.

Estrade Marc-Antoine, ThIESSET Christine, 1998, « Des débuts de carrière moins assurés », Insee Première, $\mathrm{n}^{\circ} 598,4 \mathrm{p}$.

Fondeur Yannick, MinNi Claude, 1999, «Emploi des jeunes et conjoncture », Premières informations et premières synthèses, $\mathrm{n}^{\circ} 51.1,10 \mathrm{p}$.

Galland Olivier, 1995, «Une entrée de plus en plus tardive dans la vie adulte », Économie et Statistique, $\mathrm{n}^{\circ} 283-284$, p. 33-52.

GaLland Olivier, 2000, «Entrer dans la vie adulte : des étapes toujours plus tardives mais resserrées », Économie et statistique, n $^{\circ} 337-338$.

Galland Olivier, Meron Monique, 1996, «Les frontières de la jeunesse », Données sociales 1996, p. 324-327.

HoEm J., 1985, "Weighting, Misclassification and other Issues in the Analysis of Survey Samples of Life Histories », Longitudinal Analysis of Labour Market data, Ed. J. Heckman \& B. Singer, Cambridge University Press.

Kempeneers Marianne, Lelièvre Éva, 1991, «Analyse biographique du travail féminin », European Journal of Population, $\mathrm{n}^{\circ}$ 7, p. 377-400.

Kieffer Annick, Marry Catherine, Meron Monique, Solaz Anne, «Family formation in an uncertain labour market », à paraître en 2002.

LERIDON Henri, 1985, « La baisse de la fécondité depuis 1965 : moins d'enfants désirés et moins de grossesses non désirées », Population, 40 (3), p. 507-526.

Leridon Henri, Villeneuve-GoKalP Catherine, 1994, Constance et inconstances de la famille. Biographies familiales des couples et des enfants, Ined (coll. Travaux et Documents, cahier $\left.\mathrm{n}^{\circ} 134\right), 342 \mathrm{p}$.

MARUANi Margaret (éd.), 1998, Les nouvelles frontières de l'inégalité, La Découverte, 283 p.

Meron Monique, MinNi Claude, 1995, «Des études à l'emploi : plus tard et plus difficilement qu'il y a vingt ans ", Économie et statistique, ${ }^{\circ}$ 283-284, p. 9-32.

Nicole-Drancourt Chantal, 1989, Le labyrinthe de l'insertion.

Nicole-Drancourt Chantal, Roulleau-Berger Laurence, 1995, L'insertion des jeunes en France, Paris, Puf, (coll. Que sais-je? n 2977), 127 p. 
Prioux France, 1996, «Le premier enfant: de plus en plus tard», Population, l'état des connaissances : la France, l'Europe, le Monde, Ined/La Découverte, p. 16-18.

Prioux France, 2001, «L'évolution démographique récente en France », Population, 56 (4), p. 571-610.

Singly François de, 1987, Fortune et infortune de la femme mariée : sociologie de la vie conjugale, Paris, Puf, 229 p.

Toulemon Laurent, 1994, «La place des enfants dans l'histoire des couples », Population, 49 (6), p. 1321-1346.

Toulemon Laurent, 1998, «La famille », dans Thélot Claude et Villac Michel, Politique familiale bilan et perspectives. Rapport à la ministre de l'Emploi et de la Solidarité et au ministre de l'Économie, des Finances et de l'Industrie, Paris, La Documentation française, p. 9-31.

Toulemon Laurent, LERIDON Henri, 1999, «La famille idéale : combien d'enfants, à quel âge? », Insee Première, $\mathrm{n}^{\circ} 652$.

Villeneuve-GoKalp Catherine, 1999, «La double famille des enfants de parents séparés », Population, 54 (1), p. 9-36. 


\section{Meron Monique, WidMER Isabelle.- Les femmes au chômage retardent l'arrivée du pre- mier enfant}

Le chômage, plus que les autres situations vis-à-vis de l'emploi, incite les jeunes femmes en couple sans enfant à retarder un éventuel projet de maternité. Ce résultat est établi ici pour les femmes nées entre 1952 et 1973, à partir des résultats de l'enquête Jeunes et carrières réalisée par l'Insee en 1997. Les jeunes femmes confrontées à une période de chômage ont temporairement renoncé à avoir un premier enfant plutôt que d'avancer son arrivée à la faveur du temps «libre » qui leur était imposé. En revanche, la situation de femme au foyer, surtout au tout début de la vie conjugale, correspond à des premières maternités beaucoup plus rapides. Ce résultat confirme qu'une période de chômage n'est pas assimilable à une période d'inactivité : au regard de la fécondité, les chômeuses ne sont pas des femmes au foyer.

La conjoncture économique a un impact particulièrement important sur les débuts de la vie professionnelle des jeunes au moment où ils sont susceptibles de construire une vie de famille. Or, les analyses statistiques courantes ne permettent pas d'établir un lien clair entre les fluctuations de la montée du chômage et le recul de l'âge à la première maternité. L'analyse biographique permet d'éclairer l'impact du chômage féminin sur les projets d'enfants, en faisant intervenir le temps écoulé entre les événements de l'histoire de vie des individus parmi les variables explicatives de leurs comportements.

Dans une société où la contraception permet le plus souvent de maîtriser le moment de devenir mère et où l'activité professionnelle des femmes est progressivement devenue la norme, ces constats montrent que les calendriers familiaux et les décisions des couples tiennent désormais compte des situations professionnelles vécues et anticipées par les deux conjoints.

\section{Meron Monique, Widmer Isabelle.- Las mujeres en paro posponen la llegada del pri- mer hijo}

El paro, más que otras situaciones laborales, incita a las mujeres jóvenes en pareja y sin hijos a posponer los proyectos de maternidad. Este artículo confirma este postulado para las mujeres nacidas entre 1952 y 1973 a través de los resultados de la encuesta Jóvenes y Carreras llevado a cabo por el INSEE en 1997. Las mujeres jóvenes en paro renuncian temporalmente a tener un primer hijo en lugar de anticipar su llegada para aprovechar el tiempo "libre" impuesto. La situación de ama de casa, en cambio, conlleva primeras maternidades mucho más jóvenes, especialmente al inicio de la vida en pareja. Este resultado confirma que un período de desempleo no es equiparable a un periodo de inactividad: en términos de fecundidad, las desempleadas no son amas de casa.

La coyuntura económica tiene un efecto importante sobre jóvenes que inician su carrera profesional, en el momento en que son susceptibles de crear una familia. Los análisis estadísticos existentes no permiten establecer una relación clara entre el aumento del paro y el retraso de la maternidad. El análisis biográfico, sin embargo, permite medir el impacto del paro femenino sobre los proyectos de descendencia ya que incluye el tiempo entre sucesos de la historia de vida de los individuos entre las variables explicativas de sus comportamientos.

Una sociedad en la cual los anticonceptivos permiten controlar la fecundidad y en la cual la actividad profesional de las mujeres se ha generalizado, estos resultados muestran que las situaciones profesionales presentes y futuras de ambos cónyuges influyen en los calendarios familiares y las decisiones de las parejas.

Monique Meron, Institut national d'études démographiques, 133 bd Davout, 75980 Paris Cedex 20, France, tél. : 330 (1) 56062153 , fax : 330 (1) 56062199 , courriel : meron@ined.fr 